\title{
Axisymmetric Stokes flow due to a point-force singularity acting between two coaxially positioned rigid no-slip disks
}

\author{
Abdallah Daddi-Moussa-Ider ${ }^{1}{ }^{\dagger}$, Alexander R. Sprenger ${ }^{1}$, \\ Yacine Amarouchene ${ }^{2}$, Thomas Salez ${ }^{2,3}$, Clarissa Schönecker ${ }^{4,5}$, \\ Thomas Richter ${ }^{6}$, Hartmut Löwen ${ }^{1}$ and Andreas M. Menzel ${ }^{1,7}$ \\ ${ }^{1}$ Institut für Theoretische Physik II: Weiche Materie, Heinrich-Heine-Universität Düsseldorf, \\ 40225 Düsseldorf, Germany \\ ${ }^{2}$ Univ. Bordeaux, CNRS, LOMA, UMR 5798, 33405 Talence, France \\ ${ }^{3}$ Global Station for Soft Matter, Global Institution for Collaborative Research and Education, \\ Hokkaido University, Sapporo, Hokkaido 060-0808, Japan \\ ${ }^{4}$ Technische Universität Kaiserslautern, 67663 Kaiserslautern, Germany \\ ${ }^{5}$ Max-Planck-Institut für Polymerforschung, 55218 Mainz, Germany \\ ${ }^{6}$ Institut für Analysis und Numerik, Otto-von-Guericke-Universität Magdeburg, Universitätsplatz 2, \\ 39106 Magdeburg, Germany \\ ${ }^{7}$ Institut für Physik, Otto-von-Guericke-Universität Magdeburg, Universitätsplatz 2, 39106 Magdeburg, \\ Germany
}

(Received 26 March 2020; revised 8 June 2020; accepted 14 August 2020)

We investigate theoretically, on the basis of the steady Stokes equations for a viscous incompressible fluid, the flow induced by a stokeslet located on the centre axis of two coaxially positioned rigid disks. The stokeslet is directed along the centre axis. No-slip boundary conditions are assumed to hold at the surfaces of the disks. We perform the calculation of the associated Green's function in large parts analytically, reducing the spatial evaluation of the flow field to one-dimensional integrations amenable to numerical treatment. To this end, we formulate the solution of the hydrodynamic problem for the viscous flow surrounding the two disks as a mixed boundary-value problem, which we then reduce to a system of four dual integral equations. We show the existence of viscous toroidal eddies arising in the fluid domain bounded by the two disks, manifested in the plane containing the centre axis through adjacent counter-rotating eddies. Additionally, we probe the effect of the confining disks on the slow dynamics of a point-like particle by evaluating the hydrodynamic mobility function associated with axial motion. Thereupon, we assess the appropriateness of the commonly employed superposition approximation and discuss its validity and applicability as a function of the geometrical properties of the system. Additionally, we complement our semi-analytical approach by finite-element computer simulations, which reveals a good agreement. Our results may find applications

$†$ Email address for correspondence: abdallah.daddi.moussa.ider@uni-duesseldorf.de 
in guiding the design of microparticle-based sensing devices and electrokinetic transport in small-scale capacitors.

Key words: colloids, Navier-Stokes equations

\section{Introduction}

Manipulating colloidal particles suspended in viscous media is a challenging task and is of paramount importance in various fields of engineering and natural sciences. Frequently, taking into account the fluid-mediated hydrodynamic interactions between particles moving through a liquid is essential to predict the behaviour of colloidal suspensions and polymer solutions (Probstein 2005; Mewis \& Wagner 2012). Recent advances in microand nanofluidic technologies have permitted the fabrication and manufacturing of channels with well-defined geometries and characteristic dimensions ranging from the micro- to the nanoscale. A deep understanding of the nature of the mutual interactions between particles and their confining interfaces is of crucial importance in guiding the design of devices and tools for an optimal nanoscale control of biological macromolecules. Notable examples include single-molecule manipulation (Turner et al. 1998; Campbell et al. 2004), DNA mapping for genomic applications (Reisner et al. 2005; Riehn et al. 2005; Persson \& Tegenfeldt 2010), DNA separation and sorting (Doyle et al. 2002; Cross, Strychalski \& Craighead 2007; Xia, Yan \& Hou 2012), and rheological probing of complex structures using atomic force microscopy cantilevers (François et al. 2008, 2009; Dufour et al. 2012; Darwiche et al. 2013).

At these small scales, fluid flows are governed by low-Reynolds-number hydrodynamics, where viscous effects dominate over inertial effects (Kim \& Karrila 2013). Solutions for fluid flows due to point forces, or stokeslets, acting close to confining boundaries have been tabulated for various types of geometries, as summarised in the classic textbook by Happel \& Brenner (1983). The study of the fluid-mediated hydrodynamic interactions in a channel confinement has received significant attention from many researchers over the past couple of years. In the following, we provide a survey of the current state of the art and summarise the relevant literature on this subject.

The first attempt to address the motion of a spherical particle confined between two infinitely extended no-slip walls dates back to Faxén (1921), who calculated in his $\mathrm{PhD}$ dissertation the hydrodynamic mobility parallel to the walls. These calculations were performed when the particle is located in the quarter-plane or mid-plane between the two confining walls (Happel \& Brenner 1983). Later, Oseen (1928) suggested that the hydrodynamic mobility between two walls could approximately be obtained by superposition of the contributions resulting from each single wall. A modified coherent superposition approximation was further suggested by Benesch, Yiacoumi \& Tsouris (2003), providing the diffusion coefficients of a Brownian sphere in confining channels. These predictions were found to match more accurately the existing experimental data reported in the literature.

Exact solutions for a point-force singularity acting at an arbitrary position between two walls were first obtained using the image technique in a seminal article by Liron \& Mochon (1976). It was noted that the effect of the second wall becomes important when the distance separating the particle from the closest wall is larger than approximately one-tenth of the channel width (Brenner 1999). Using this solution, Liron (1978) further investigated the fluid transport problem of cilia between two parallel plates. 
A joint analytical-numerical approach (Ganatos, Pfeffer \& Weinbaum 1980a; Ganatos, Weinbaum \& Pfeffer 1980b) as well as a multipole expansion technique (Swan \& Brady 2010) were presented to address the motion of an extended particle confined between two hard walls. Bhattacharya \& Bławzdziewicz (2002) constructed the image system for the flow field produced by a force multipole in a space bounded by two parallel walls using the image representation for Stokes flow. In addition, compressibility effects were examined by Felderhof $(2006,2010 a, b)$. In this context, Hackborn (1990) investigated the asymmetric Stokes flow between two parallel planes due to a rotlet singularity, the axis of which is parallel to the boundary planes. Further, Ozarkar \& Sangani (2008) prescribed an analytical approach using the image-system technique for determining the Stokes flow around particles in a thin film bounded by a wall and a gas-liquid interface. More recently, Daddi-Moussa-Ider, Guckenberger \& Gekle (2016) provided the frequency-dependent hydrodynamic mobility functions between two planar elastic interfaces endowed with resistance towards shear and bending deformation modes.

Experimentally, Dufresne, Altman \& Grier (2001) reported direct imaging measurements of a colloidal particle diffusing between two parallel surfaces, finding a good agreement with the superposition approximation suggested by Oseen. In addition, video microscopy (Faucheux \& Libchaber 1994) combined with optical tweezers (Lin, Yu \& Rice 2000; Tränkle, Ruh \& Rohrbach 2016) as well as dynamic light scattering (Lobry \& Ostrowsky 1996) have also allowed for good agreement with available theoretical predictions. Further experimental investigations have focused on DNA conformation and diffusion in slit-like confinements (Balducci et al. 2006; Stein et al. 2006; Strychalski, Levy \& Craighead 2008; Tang et al. 2010; Graham 2011; Dai et al. 2013; Jones, van der Maarel \& Doyle 2013).

Concerning collective properties, the behaviour of suspensions in a channel bounded by two planar walls has received a lot of attention. For instance, Bhattacharya, Bławzdziewicz \& Wajnryb (2005) examined the fluid-mediated hydrodynamic interactions in a suspension of spherical particles confined between two parallel planar walls under creeping-flow conditions. In addition, Bhattacharya (2008) considered the collective motion of a two-dimensional periodic array of colloidal particles in a slit pore. Using a novel accelerated Stokesian-dynamics algorithm, Baron, Bławzdziewicz \& Wajnryb (2008) performed fully resolved computer simulations to investigate the collective motion of linear trains and regular square arrays of particles suspended in a viscous fluid bounded by two parallel plates. Further, Bławzdziewicz \& Wajnryb (2008) analysed the far-field response to external forcing of a suspension of particles in a channel. Swan \& Brady (2011) presented a numerical method for computing the hydrodynamic forces exerted on particles in a suspension confined between two parallel walls. Furthermore, Saintillan, Shaqfeh \& Darve (2006) employed Brownian dynamics simulations to investigate the effect of chain flexibility on the cross-streamline migration of short polymers in a pressure-driven flow between two flat plates. The latter numerical study confirmed the existence of a shear-induced migration towards the channel centreline away from the confining solid boundaries.

The hydrodynamic problem of particles freely moving between plane-parallel walls in the presence of an incident flow has been further considered in still more details. Under an external flow, Uspal, Eral \& Doyle (2013) showed how shape and geometric confinement of rigid microparticles can conveniently be tailored for self-steering. Jones (2004) made use of a two-dimensional Fourier-transform technique to obtain an analytic expression of the Green tensor for the Stokes equations with an incident Poiseuille flow. In addition, he provided the elements of the resistance and mobility tensors in this slit-like geometry. Bhattacharya, Bławzdziewicz \& Wajnryb (2006) introduced a novel numerical algorithm 
based on transformations between Cartesian and spherical representations of Stokes flow to account for an incident Poiseuille flow. Staben, Zinchenko \& Davis (2003) presented a novel boundary-integral algorithm for the motion of a particle between two parallel planar walls in Poiseuille flow. The boundary-integral method formulated in their work allowed the effects of the confining walls to be directly incorporated into the stress tensor, without requiring discretisation of the two walls. In this context, Griggs, Zinchenko \& Davis (2007) and Janssen \& Anderson $(2007,2008)$ employed boundary-integral methods to examine the motion of a deformable drop between two parallel walls in Poiseuille flow, where lateral migration towards the channel centre is observed.

Geometric confinement significantly alters the behaviour of swimming micro-organisms and can affect the motility of self-propelling active particles in a pronounced way (Lauga \& Powers 2009; Menzel 2013, 2015; Bechinger et al. 2016; Lauga 2016; Zöttl \& Stark 2016; Ostapenko et al. 2018; Gompper et al. 2020; Shaebani et al. 2020). Surface-related effects on microswimmers can lead to crucial implications for biofilm formation and microbial activity. In a channel bounded by two walls, Bilbao et al. (2013) studied the locomotion of a model nematode, finding that the swimming organism tends to swim faster and navigate more effectively under confinement. Furthermore, Wu et al. $(2015,2016)$ investigated the effect of confinement on the swimming behaviour of a model eukaryotic cell undergoing amoeboid motion. There, the swimmer has been modelled as an inextensible membrane deploying local active force. It has been found that confinement can strongly alter the swimming gait. In addition, Brotto et al. (2013) described theoretically the dynamics of self-propelling active particles in rigidly confined thin liquid films. They demonstrated that, due to hydrodynamic friction with the nearby rigid walls, confined microswimmers not only reorient themselves in response to flow gradients but also can show reorientation in uniform flows. In this context, Mathijssen et al. (2016) investigated theoretically the hydrodynamics of self-propelling microswimmers in a thin film. Daddi-Moussa-Ider et al. (2018) examined the behaviour of a three-sphere microswimmer in a channel bounded by two walls, where different swimming states have been observed. More recently, amoeboid swimming in a compliant channel was numerically investigated (Dalal, Farutin \& Misbah 2020).

In all of the above-mentioned studies, the confining channel was assumed to be of infinite extent or periodically replicated along the lateral directions. Instead, here we consider the hydrodynamic problem for a point force acting near two coaxially positioned disks of finite radius. In many biologically and industrially relevant applications, finite-size effects become crucial for an accurate and reliable description of transport processes ranging from the microscale to the nanoscale. Prime examples include the ionic transport and electrokinetics in small-scale capacitors (Marini Bettolo Marconi \& Melchionna 2012; Thakore \& Hickman 2015; Babel, Eikerling \& Löwen 2018; Asta et al. 2019), electrochemomechanical energy conversion in microfluidic channels (Daiguji et al. 2004), and the rheology of droplets, capsules or cells in constricted/structured microchannels (Park \& Dimitrakopoulos 2013; Le Goff et al. 2017; Trégouët et al. 2018, 2019), where boundary effects may play a pivotal role.

In this paper, we take a step towards addressing this context by presenting an analytical theory for the viscous flow resulting from a stokeslet singularity acting along the centre axis of two coaxially positioned disks of no-slip surfaces. We formulate the hydrodynamic problem as a mixed boundary-value problem, which we then transform into a system of dual integral equations. Along this path, we show that the solution of the flow field in the fluid region bounded by the two disks exhibits viscous toroidal eddies. In addition to that, we derive expressions for the hydrodynamics mobility functions and discuss the applicability and limitations of the superposition approximation. Moreover, we support 


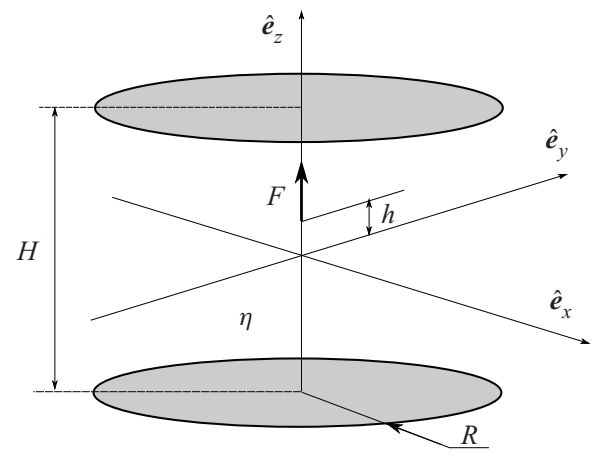

FIGURE 1. Schematic of the system. The surrounding viscous Newtonian fluid is set into motion through the action of a point-force singularity located on the symmetry axis of two coaxially positioned disks.

our semi-analytical results by numerical simulations using a finite-element method (FEM), which leads to a good agreement.

The remainder of this paper is organised as follows. In $\S 2$, we formulate the problem mathematically and derive the corresponding system of dual integral equations, from which the solution for the hydrodynamic flow fields can be obtained. We then make use of this solution in $\S 3$ to yield an integral expression of the mobility function of a point-like particle slowly translating along the axis of the disks. Concluding remarks and outlooks are contained in $\S 4$. In appendix A, we detail the analytical derivation of the kernel functions arising in the resulting integral equations.

\section{Mathematical formulation}

We examine the axisymmetric flow induced by a stokeslet singularity acting on the axis of symmetry of two coaxially positioned circular disks of equal radius $R$. Moreover, we suppose that the disks are located within the planes $z=-H / 2$ and $z=H / 2$, with $H$ denoting the separation distance between the disks. Their centres are positioned on the $z$ axis. In addition, we assume that the surrounding viscous fluid is Newtonian, of constant dynamic viscosity $\eta$, and that the flow is incompressible.

\subsection{Governing equations}

In low-Reynolds-number hydrodynamics, the fluid dynamics is governed by the Stokes equations (Happel \& Brenner 1983)

$$
\begin{gathered}
\boldsymbol{\nabla} \cdot \boldsymbol{v}=0, \\
\nabla \cdot \boldsymbol{\sigma}+F \delta\left(\boldsymbol{r}-\boldsymbol{r}_{0}\right) \hat{\boldsymbol{e}}_{z}=\mathbf{0},
\end{gathered}
$$

where $v$ and $\sigma$ denote, respectively, the fluid velocity field and the hydrodynamic stress tensor. For a Newtonian fluid, the latter is given by $\sigma=-p \boldsymbol{I}+2 \eta \boldsymbol{E}$, where $p$ is the pressure field and $\boldsymbol{E}=\left(\boldsymbol{\nabla} \boldsymbol{v}+(\boldsymbol{\nabla})^{\mathrm{T}}\right) / 2$ is the rate-of-strain tensor, with the superscript $\mathrm{T}$ denoting a transpose. In addition, $\delta$ stands for the Dirac delta function, and $F$ is the amplitude of a stationary point force acting on the fluid at position $\boldsymbol{r}_{0}=h \hat{\boldsymbol{e}}_{z}$, where $-H / 2<h<H / 2$, with $\hat{\boldsymbol{e}}_{z}$ denoting the unit vector along the $z$ direction. See figure 1 for an illustration of the system set-up. In the remainder of this paper, we scale all the lengths involved in the problem by the separation $H$ of the two disks. 
We designate by the subscript 1 the variables and parameters in the fluid region underneath the plane containing the lower disk, for which $z \leq-1 / 2$, by the subscript 2 the fluid domain bounded by the planes $z=-1 / 2$ and $z=1 / 2$, and by the subscript 3 the region above the plane containing the upper disk, for which $z \geq 1 / 2$. Since the system is axisymmetric, all field variables are thus functions of the radial and axial coordinates only. Accordingly, the Stokes equations (2.1) can be projected onto the cylindrical coordinate system as

$$
\begin{gathered}
\frac{v_{r}}{r}+\frac{\partial v_{r}}{\partial r}+\frac{\partial v_{z}}{\partial z}=0, \\
-\frac{\partial p}{\partial r}+\eta\left(\Delta v_{r}-\frac{v_{r}}{r^{2}}\right)=0, \\
-\frac{\partial p}{\partial z}+\eta \Delta v_{z}+F \delta\left(\boldsymbol{r}-\boldsymbol{r}_{0}\right)=0,
\end{gathered}
$$

wherein $v_{r}$ and $v_{z}$ denote the radial and axial fluid velocities, respectively, and $\Delta$ is the Laplace operator given by

$$
\Delta:=\frac{\partial^{2}}{\partial r^{2}}+\frac{1}{r} \frac{\partial}{\partial r}+\frac{\partial^{2}}{\partial z^{2}} .
$$

We note that the three-dimensional Dirac delta function is expressed in axisymmetric cylindrical coordinates as $\delta\left(\boldsymbol{r}-\boldsymbol{r}_{0}\right)=(\pi r)^{-1} \delta(r) \delta(z-h)$ (Bracewell 1999).

In an unbounded viscous fluid, i.e. in the absence of the disks, the solution of equations (2.2) is given by the Oseen tensor, commonly denominated as the free-space Green function (Kim \& Karrila 2013)

$$
v_{r}^{S}=\frac{F}{8 \pi \eta} \frac{r(z-h)}{\rho^{3}}, \quad v_{z}^{S}=\frac{F}{8 \pi \eta}\left(\frac{2}{\rho}-\frac{r^{2}}{\rho^{3}}\right),
$$

with the distance from the position of the point force $\rho=\left(r^{2}+(z-h)^{2}\right)^{1 / 2}$. The corresponding pressure field reads

$$
p^{S}=\frac{F}{4 \pi} \frac{z-h}{\rho^{3}} .
$$

In the presence of the confining disks, the solution of the flow problem can be expressed as a superposition of the solution in an unbounded fluid, given above by $(2.4 a, b)$ and $(2.5)$, and a complementary solution, the sum of the two solutions being required to satisfy the underlying regularity and boundary conditions. Then

$$
\boldsymbol{v}=\boldsymbol{v}^{S}+\boldsymbol{v}^{*}, \quad p=p^{S}+p^{*},
$$

wherein $\boldsymbol{v}^{*}$ and $p^{*}$ stand for the complementary solutions (also referred to as the image solution (Blake 1971)) for the velocity and pressure fields, respectively.

For an axisymmetric Stokes flow, the general solution can be expressed in terms of two harmonic functions $\phi$ and $\psi$ as (Imai 1973; Kim 1983)

$$
v_{r}^{*}=z \frac{\partial \phi}{\partial r}+\frac{\partial \psi}{\partial r}, \quad v_{z}^{*}=z \frac{\partial \phi}{\partial z}-\phi+\frac{\partial \psi}{\partial z}, \quad p^{*}=2 \eta \frac{\partial \phi}{\partial z},
$$

with

$$
\Delta \phi=0, \quad \Delta \psi=0 .
$$


In each of the three fluid domains introduced above, the solution of Laplace's equations $(2.8 a, b)$ can be expressed in terms of Fourier-Bessel integrals as

$$
\begin{aligned}
& \phi_{i}=\frac{F}{8 \pi \eta} \int_{0}^{\infty}\left(A_{i}^{+}(\lambda) \mathrm{e}^{\lambda z}+A_{i}^{-}(\lambda) \mathrm{e}^{-\lambda z}\right) J_{0}(\lambda r) \mathrm{d} \lambda, \\
& \psi_{i}=\frac{F}{8 \pi \eta} \int_{0}^{\infty}\left(B_{i}^{+}(\lambda) \mathrm{e}^{\lambda z}+B_{i}^{-}(\lambda) \mathrm{e}^{-\lambda z}\right) J_{0}(\lambda r) \mathrm{d} \lambda,
\end{aligned}
$$

for $i \in\{1,2,3\}$, with $\lambda$ denoting the wavenumber and $J_{k}$ the $k$ th-order Bessel function of the first kind (Abramowitz \& Stegun 1972). In addition, $A_{i}^{ \pm}$and $B_{i}^{ \pm}$are wavenumber-dependent unknown coefficients, to be determined from the regularity and boundary conditions. Then, the components of the image velocity and pressure fields are given by

$$
\begin{gathered}
v_{r i}^{*}=-\frac{F}{8 \pi \eta} \int_{0}^{\infty} \lambda\left(\left(z A_{i}^{+}+B_{i}^{+}\right) \mathrm{e}^{\lambda z}+\left(z A_{i}^{-}+B_{i}^{-}\right) \mathrm{e}^{-\lambda z}\right) J_{1}(\lambda r) \mathrm{d} \lambda, \\
v_{z i}^{*}=-\frac{F}{8 \pi \eta} \int_{0}^{\infty}\left(E_{i}^{+} \mathrm{e}^{\lambda z}+E_{i}^{-} \mathrm{e}^{-\lambda z}\right) J_{0}(\lambda r) \mathrm{d} \lambda, \\
p_{i}^{*}=\frac{F}{4 \pi} \int_{0}^{\infty} \lambda\left(A_{i}^{+} \mathrm{e}^{\lambda z}-A_{i}^{-} \mathrm{e}^{-\lambda z}\right) J_{0}(\lambda r) \mathrm{d} \lambda,
\end{gathered}
$$

for $i \in\{1,2,3\}$, where we have defined the abbreviations $E_{i}^{ \pm}=(1 \mp \lambda z) A_{i}^{ \pm} \mp \lambda B_{i}^{ \pm}$.

\subsection{Boundary conditions and dual integral equations}

As regularity conditions, for the image field we require the velocity and pressure far away from the singularity location to vanish as $\rho \rightarrow \infty$. This implies that $A_{1}^{-}=B_{1}^{-}=A_{3}^{+}=$ $B_{3}^{+}=0$. In what follows, to simplify notation, we drop the plus sign in the fluid domain underneath the lower disk to denote $A_{1}=A_{1}^{+}$and $B_{1}=B_{1}^{+}$, and we drop the minus sign in the fluid domain above the upper disk to denote $A_{3}=A_{3}^{-}$and $B_{3}=B_{3}^{-}$.

The boundary conditions consist of requiring $(a)$ the natural continuity of the total fluid velocity field at the interfaces between the fluid domains, $(b)$ vanishing total velocities at the surfaces of the disks (the no-slip and no-permeability boundary condition Lauga, Brenner \& Stone 2007), and (c) continuity of the total viscous-stress vectors at the interfaces between the fluid domains outside the regions occupied by the disks. Mathematically, these conditions can be expressed as

$$
\begin{gathered}
\left.\left(\boldsymbol{v}_{1}-\boldsymbol{v}_{2}\right)\right|_{z=-1 / 2}=\left.\left(\boldsymbol{v}_{2}-\boldsymbol{v}_{3}\right)\right|_{z=1 / 2}=\mathbf{0} \quad(r>0), \\
\left.\boldsymbol{v}_{1}\right|_{z=-1 / 2}=\left.\boldsymbol{v}_{2}\right|_{z= \pm 1 / 2}=\left.\boldsymbol{v}_{3}\right|_{z=1 / 2}=\mathbf{0} \quad(r<R), \\
\left.\left(\boldsymbol{\sigma}_{2}-\boldsymbol{\sigma}_{1}\right) \cdot \hat{\boldsymbol{e}}_{z}\right|_{z=-1 / 2}=\left.\left(\boldsymbol{\sigma}_{3}-\boldsymbol{\sigma}_{2}\right) \cdot \hat{\boldsymbol{e}}_{z}\right|_{z=1 / 2}=\mathbf{0} \quad(r>R),
\end{gathered}
$$

where the components of the stress vector are expressed in cylindrical coordinates for an axisymmetric flow field by

$$
\boldsymbol{\sigma}_{i} \cdot \hat{\boldsymbol{e}}_{z}=\eta\left(\frac{\partial v_{r i}}{\partial z}+\frac{\partial v_{z i}}{\partial r}\right) \hat{\boldsymbol{e}}_{r}+\left(-p_{i}+2 \eta \frac{\partial v_{z i}}{\partial z}\right) \hat{\boldsymbol{e}}_{z}, \quad i \in\{1,2,3\} .
$$

Applying the continuity of the radial components of the fluid velocity at the surfaces occupied by the two disks yields the expressions of the wavenumber-dependent 
coefficients associated with the intermediate fluid domain bounded by the two disks as functions of those in the lower and upper fluid domains. Defining $X_{2}=\left(A_{2}^{-}, B_{2}^{-}, A_{2}^{+}, B_{2}^{+}\right)^{\mathrm{T}}$ and $X_{13}=\left(A_{1}, B_{1}, A_{3}, B_{3}\right)^{\mathrm{T}}$, we obtain

$$
X_{2}=Q \cdot X_{13},
$$

where the matrix $\boldsymbol{Q}$ is given by

$$
\boldsymbol{Q}=\left(s^{2}-\lambda^{2}\right)^{-1}\left(\begin{array}{cccc}
\frac{1}{2}(s+\lambda c) & -\lambda s & -\frac{1}{2} \phi^{+} & -\lambda^{2} \\
\frac{1}{4} \lambda s & \frac{1}{2}(s-\lambda c) & -\frac{1}{4} \lambda^{2} & -\frac{1}{2} \phi^{-} \\
-\frac{1}{2} \phi^{+} & \lambda^{2} & \frac{1}{2}(s+\lambda c) & \lambda s \\
\frac{1}{4} \lambda^{2} & -\frac{1}{2} \phi^{-} & -\frac{1}{4} \lambda s & \frac{1}{2}(s-\lambda c)
\end{array}\right) .
$$

Here, we have defined for convenience the abbreviations $s=\sinh (\lambda)$ and $c=\cosh (\lambda)$. In addition, $\phi^{ \pm}=\lambda(\lambda \pm 1)+s \mathrm{e}^{-\lambda}$.

On the one hand, by addressing the no-slip velocity boundary conditions at the surfaces of the disks prescribed by $(2.11 b)$ and projecting the resulting equations onto the radial and tangential directions, four integral equations on the inner domain are obtained:

$$
\begin{gathered}
\int_{0}^{\infty} \lambda\left(\frac{1}{2} A_{1}-B_{1}\right) \mathrm{e}^{-(\lambda / 2)} J_{1}(\lambda r) \mathrm{d} \lambda=\psi_{1}^{+}(r) \quad(r<R), \\
\int_{0}^{\infty} \lambda\left(\frac{1}{2} A_{3}+B_{3}\right) \mathrm{e}^{-(\lambda / 2)} J_{1}(\lambda r) \mathrm{d} \lambda=\psi_{1}^{-}(r) \quad(r<R), \\
\int_{0}^{\infty}\left(A_{1}+\lambda\left(\frac{1}{2} A_{1}-B_{1}\right)\right) \mathrm{e}^{-(\lambda / 2)} J_{0}(\lambda r) \mathrm{d} \lambda=\psi_{2}^{+}(r) \quad(r<R), \\
\int_{0}^{\infty}\left(A_{3}+\lambda\left(\frac{1}{2} A_{3}+B_{3}\right)\right) \mathrm{e}^{-(\lambda / 2)} J_{0}(\lambda r) \mathrm{d} \lambda=\psi_{2}^{-}(r) \quad(r<R) .
\end{gathered}
$$

Here the terms appearing on the right-hand sides in these equations are radial functions resulting from the evaluation of the terms associated with the flow velocity field induced by the free-space stokeslet at the surfaces of the coaxially positioned disks. They are explicitly given by

$$
\psi_{1}^{ \pm}(r)=\frac{ \pm r\left(h \pm \frac{1}{2}\right)}{\left(r^{2}+\left(h \pm \frac{1}{2}\right)^{2}\right)^{3 / 2}}, \quad \psi_{2}^{ \pm}(r)=\frac{r^{2}+2\left(h \pm \frac{1}{2}\right)^{2}}{\left(r^{2}+\left(h \pm \frac{1}{2}\right)^{2}\right)^{3 / 2}} .
$$

On the other hand, four integral equations on the outer domain are obtained by addressing the continuity of the hydrodynamic stress vector at $z= \pm 1 / 2$ prescribed by (2.11c). They can be cast in the form

$$
\begin{array}{ll}
\int_{0}^{\infty} g_{i}(\lambda) J_{1}(\lambda r) \mathrm{d} \lambda=0 & (r>R), \quad i \in\{1,3\}, \\
\int_{0}^{\infty} g_{i}(\lambda) J_{0}(\lambda r) \mathrm{d} \lambda=0 & (r>R), \quad i \in\{2,4\},
\end{array}
$$


where we have defined the wavenumber-dependent quantities

$$
\begin{gathered}
g_{1}(\lambda)=\lambda^{2}\left(\left(\frac{1}{2} A_{2}^{-}-B_{2}^{-}\right) \mathrm{e}^{\lambda / 2}+\left(\frac{1}{2}\left(A_{1}-A_{2}^{+}\right)+B_{2}^{+}-B_{1}\right) \mathrm{e}^{-(\lambda / 2)}\right), \\
g_{3}(\lambda)=\lambda^{2}\left(\left(\frac{1}{2} A_{2}^{+}+B_{2}^{+}\right) \mathrm{e}^{\lambda / 2}+\left(\frac{1}{2}\left(A_{3}-A_{2}^{-}\right)+B_{3}-B_{2}^{-}\right) \mathrm{e}^{-(\lambda / 2)}\right), \\
g_{2}(\lambda)=C^{-} \mathrm{e}^{\lambda / 2}+\lambda\left(\left(1+\frac{1}{2} \lambda\right)\left(A_{1}-A_{2}^{+}\right)+\lambda\left(B_{2}^{+}-B_{1}\right)\right) \mathrm{e}^{-(\lambda / 2)}, \\
g_{4}(\lambda)=C^{+} \mathrm{e}^{\lambda / 2}+\lambda\left(\left(1+\frac{1}{2} \lambda\right)\left(A_{3}-A_{2}^{-}\right)+\lambda\left(B_{3}-B_{2}^{-}\right)\right) \mathrm{e}^{-(\lambda / 2)},
\end{gathered}
$$

wherein $C^{ \pm}=\lambda\left((1-\lambda / 2) A_{2}^{ \pm} \mp \lambda B_{2}^{ \pm}\right)$.

Inserting (2.13) and (2.14), equations (2.15)-(2.18) form a system of four dual integral equations (Tricomi 1985) for the unknown wavenumber-dependent coefficients regrouped in $X_{13}$. A solution of such types of dual integral equations with Bessel kernels can be obtained by the methods prescribed by Sneddon $(1960,1966)$ and Copson (1961). A similar procedure has recently been employed by some of us to address the axisymmetric flow induced by a stokeslet near a circular elastic membrane (Daddi-Moussa-Ider, Kaoui \& Löwen 2019), and the asymmetric flow field near a finite-sized rigid disk (Daddi-Moussa-Ider et al. 2020). Once $X_{13}$ is determined from solving the dual integral equations derived above, the remaining wavenumber-dependent coefficients expressed by $X_{2}$ follow forthwith from (2.13) and (2.14).

The core idea of our solution approach consists of expressing the solution of (2.17) as definite integrals of the forms

$$
g_{i}(\lambda)=2 \lambda^{1 / 2} \int_{0}^{R} f_{i}(t) J_{3 / 2}(\lambda t) \mathrm{d} t, \quad i \in\{1,3\},
$$

and

$$
g_{i}(\lambda)=2 \lambda^{1 / 2} \int_{0}^{R} f_{i}(t) J_{1 / 2}(\lambda t) \mathrm{d} t, \quad i \in\{2,4\},
$$

where $f_{i}:[0, R] \rightarrow \mathbb{R}$, for $i \in\{1,2,3,4\}$, are unknown functions to be determined. Accordingly, the integral equations in the outer domain boundaries are automatically satisfied upon making use of the following identity, which holds for any positive integer $p$ (Abramowitz \& Stegun 1972),

$$
\int_{0}^{\infty} \lambda^{1 / 2} J_{p}(\lambda r) J_{p+1 / 2}(\lambda t) \mathrm{d} \lambda=0 \quad(0<t<r) .
$$

By solving (2.18) for the coefficients $A_{1}, B_{1}, A_{3}$ and $B_{3}$ upon making use of (2.13) and (2.14), equation (2.15) can be rewritten as

$$
\begin{aligned}
& \int_{0}^{\infty}(2 \lambda)^{-1}\left(g_{1}(\lambda)+(\lambda-1) \mathrm{e}^{-\lambda} g_{3}(\lambda)+\lambda \mathrm{e}^{-\lambda} g_{4}(\lambda)\right) J_{1}(\lambda r) \mathrm{d} \lambda=\psi_{1}^{+}(r), \\
& \int_{0}^{\infty}(2 \lambda)^{-1}\left((\lambda-1) \mathrm{e}^{-\lambda} g_{1}(\lambda)+\lambda \mathrm{e}^{-\lambda} g_{2}(\lambda)+g_{3}(\lambda)\right) J_{1}(\lambda r) \mathrm{d} \lambda=\psi_{1}^{-}(r), \\
& \int_{0}^{\infty}(2 \lambda)^{-1}\left(g_{2}(\lambda)+\lambda \mathrm{e}^{-\lambda} g_{3}(\lambda)+(\lambda+1) \mathrm{e}^{-\lambda} g_{4}(\lambda)\right) J_{0}(\lambda r) \mathrm{d} \lambda=\psi_{2}^{+}(r), \\
& \int_{0}^{\infty}(2 \lambda)^{-1}\left(\lambda \mathrm{e}^{-\lambda} g_{1}(\lambda)+(\lambda+1) \mathrm{e}^{-\lambda} g_{2}(\lambda)+g_{4}(\lambda)\right) J_{0}(\lambda r) \mathrm{d} \lambda=\psi_{2}^{-}(r) .
\end{aligned}
$$


Next, by substituting (2.19) into (2.21) and interchanging the order of the integrations with respect to the variables $t$ and $\lambda$, the equations associated with the inner problem can be expressed in the following final forms:

$$
\begin{gathered}
\int_{0}^{R}\left(L_{5}(r, t) f_{1}(t)+L_{4}(r, t) f_{3}(t)+L_{1}(r, t) f_{4}(t)\right) \mathrm{d} t=\psi_{1}^{+}(r), \\
\int_{0}^{R}\left(L_{4}(r, t) f_{1}(t)+L_{1}(r, t) f_{2}(t)+L_{5}(r, t) f_{3}(t)\right) \mathrm{d} t=\psi_{1}^{-}(r), \\
\int_{0}^{R}\left(L_{6}(r, t) f_{2}(t)+L_{3}(r, t) f_{3}(t)+L_{2}(r, t) f_{4}(t)\right) \mathrm{d} t=\psi_{2}^{+}(r), \\
\int_{0}^{R}\left(L_{3}(r, t) f_{1}(t)+L_{2}(r, t) f_{2}(t)+L_{6}(r, t) f_{4}(t)\right) \mathrm{d} t=\psi_{2}^{-}(r),
\end{gathered}
$$

where the kernels $L_{i}:[0, R]^{2} \rightarrow \mathbb{R}$, for $i \in\{1,2,3,4\}$, are complex mathematical functions that are defined and provided in appendix A.

Equations (2.22) form a system of four Fredholm integral equations of the first kind (Smithies 1958; Polyanin \& Manzhirov 1998) for the unknown functions $f_{i}(t)$, $i \in\{1,2,3,4\}$. Owing to the complicated nature of the kernel functions, we make recourse to numerical solutions.

\subsection{Numerical solution of the integral equations and comparison with FEM simulations}

We now summarise the main steps involved in the numerical computations of the flow field. First, the integration over the intervals $[0, R]$ in (2.22) are partitioned into $N$ subintervals and each integral is approximated by the standard middle Riemann sum (Davis \& Rabinowitz 2007). The four resulting equations are evaluated at $N$ values of $t_{j}$ that are uniformly distributed over the interval $[0, R]$ such that $t_{j}=(j-1 / 2)(R / N)$, with $j=1, \ldots, N$. Secondly, the discrete values of $f_{i}\left(t_{j}\right)$, with $i \in\{1,2,3,4\}$, are obtained by solving the resulting linear system of $4 N$ equations. Thirdly, the four integrals in (2.19) are converted into well-behaved definite integrals over $[0, \pi / 2]$ by using the change of variable $\lambda=\tan u$ and thus $\mathrm{d} \lambda=\mathrm{d} u / \cos ^{2} u$. Thereupon, the resulting integrals are also approximated by the middle Riemann sum, and the wavenumber-dependent functions $g_{i}\left(\lambda_{k}=\tan u_{k}\right), k=1, \ldots, M$, are evaluated at discrete values of $u_{k}$ such that $u_{k}=(k-1 / 2)(\pi / 2) / M$. Fourthly, the values of $\boldsymbol{X}_{2}$ at each discrete point $\lambda_{k}$ are readily obtained by inverting the linear system of four equations given by (2.18). In addition, it follows from (2.13) that $X_{13}=Q^{-1} \cdot X_{2}$. Finally, the image flow fields are obtained from (2.10) by approximating, again, the integrals by the middle Riemann sum.

Even though the approach employed here may seem cumbersome at first glance, it has the advantage of being amenable to straightforward implementation. Unlike many direct numerical simulation techniques, which generally require discretisation of the entire three-dimensional fluid domain, or of at least an effectively two-dimensional domain when the axial symmetry is exploited, the integral formulation presented in this work reduces the solution of the flow problem to a set of one-dimensional integrals. Besides, the present semi-analytical approach might serve as a motivation for various theoretical investigations of related problems that could possibly pave the way towards real engineering applications.

In figure 2, we present a $\log -\log$ plot of the variations of the discretisation error (Roy 2010) associated with the numerical computation of the amplitude of the image velocity 


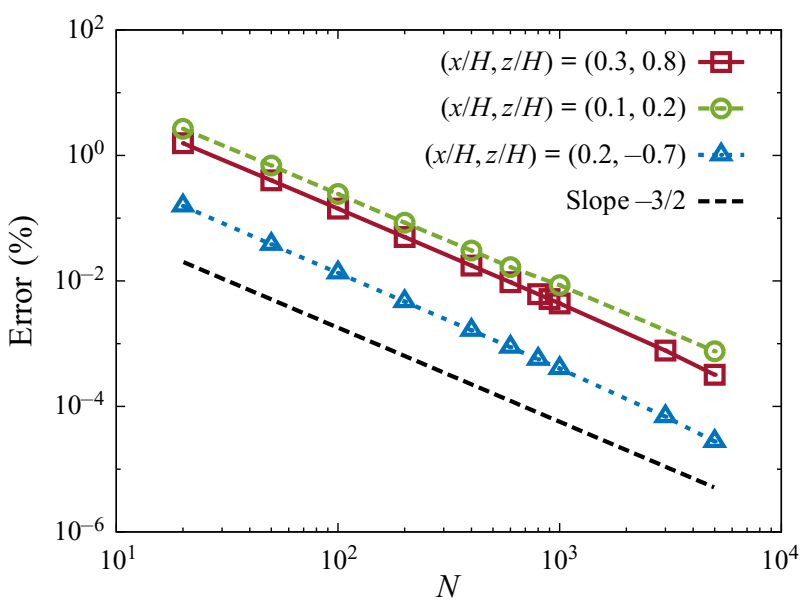

FIGURE 2. Log-log plot of the relative discretisation error occurring in the computation of the amplitude of the image velocity field versus the number of discretisation points, evaluated at various positions within the fluid domain. Here, we set $R=H, h / H=0.3$ and $M=10 N$. The errors are estimated relative to the corresponding values computed using a finer grid spacing with $N=15000$ and $M=150000$.

field versus the number of discrete points used in the numerical integration of (2.22) while keeping $M=10 N$ in the discretisation of (2.19) and (2.10). The error is estimated relative to the numerical solution on a finer gird size for $N=15000$ and $M=150000$ at three different points of the fluid domain. We observe that the error decays approximately algebraically as $N^{-3 / 2}$ over the whole range of considered values of $N$ and lies well below $10^{-3} \%$ for $N \geq 5000$. We have checked that a similar behaviour is also found when varying the position of the stokeslet or the evaluation point within the fluid domain.

To validate our semi-analytical solution, we perform direct numerical simulations for the same geometry as well. We use a piecewise-quadratic finite-element discretisation of the Stokes problem stated by (2.2) in cylindrical coordinates. Since such an equal-order discretisation does not satisfy the inf-sup condition, we add stabilisation terms of local projection type (Becker \& Braack 2001). The numerical domain is artificially limited to $(0, R) \times(-Z, Z)$ with $R, Z \in \mathbb{R}$ being sufficiently large numbers so as to avoid spurious feedback to the region of interest close to the plates. In addition, the Dirac delta function forcing the flow is represented exactly in the variational formulation by means of

$$
\int_{0}^{R} \int_{-Z}^{Z} r \delta\left(\boldsymbol{r}-\boldsymbol{r}_{0}\right) \phi_{z}(\boldsymbol{r}) \mathrm{d} r \mathrm{~d} z=\phi_{z}\left(\boldsymbol{r}_{0}\right),
$$

where $\phi_{z}$ is the test function corresponding to the vertical direction. Numerically, the singularity calls for very fine mesh resolution close to $\boldsymbol{r}_{0}$ and in proximity to the coaxially positioned plates, which we accomplish by local mesh adaptivity (Braack \& Richter 2006). Further details on the discretisation method and the solution of the resulting linear systems of equations can be found in Richter (2017).

In figure 3, we represent the graphs of the resulting streamlines as well as contour plots of the total velocity field resulting from a stokeslet singularity axisymmetrically acting at various positions along the axis of two coaxially disposed disks of unit radius. Here, we set the numbers of discrete points to $N=15000$ and $M=150000$ in our numerical evaluation of the analytical description. The magnitude of the scaled velocity field is 
shown on a logarithmic scale in order to better appreciate the difference in magnitude between the different fluid regions. In each panel, we depict on the left-hand side the results obtained via our semi-analytical approach derived in the present work. On the right-hand side in each panel, we include the corresponding flow fields determined via the FEM simulations. Good agreement between the two solution procedures is obtained over the whole fluid domain, demonstrating the robustness and applicability of our semi-analytical approach. Most noticeably, we observe the existence of adjacent counter-rotating eddies, the axis of rotation of which is directed along the azimuthal direction. Accordingly, the resulting flow field in the inner region consists of toroidal eddies on account of the axisymmetric nature of the flow (Moffatt 1964). In contrast to that, descending streamlines are obtained in the outer region. For infinitely large disks, analogous toroidal structures have previously been identified and proven to decay exponentially with distance from the singularity position (Liron \& Blake 1981). Moreover, we remark that the overall magnitude of the flow field becomes less important as the point force gets closer to a confining plate. This behaviour is accompanied by a notable increase of the asymmetry of the counter-rotating eddies.

Having derived the solution of the flow problem due to an axisymmetric stokeslet acting near two finite-sized coaxially positioned disks, we next employ our formalism to recover the solution earlier obtained by Liron \& Mochon (1976) for a stokeslet acting between two parallel planar walls of infinite extent along the transverse direction.

\subsection{Solution for $R \rightarrow \infty$}

For infinitely large disks, the integral equations (2.21) in the inner domain become defined for the whole axis of positive real numbers. Accordingly, the solution for the unknown functions $g_{i}(\lambda)$, for $i \in\{1,2,3,4\}$, can be obtained using inverse Hankel transforms. By making use of the orthogonality property of Bessel functions (Abramowitz \& Stegun 1972)

$$
\int_{0}^{\infty} r J_{v}(\lambda r) J_{v}\left(\lambda^{\prime} r\right) \mathrm{d} r=\lambda^{-1} \delta\left(\lambda-\lambda^{\prime}\right)
$$

we readily obtain

$$
\boldsymbol{H} \cdot \boldsymbol{g}=\hat{\boldsymbol{\psi}},
$$

where we have defined the unknown vector $g=\left(g_{1}, g_{2}, g_{3}, g_{4}\right)^{\mathrm{T}}$, the wavenumber-dependent matrix

$$
\boldsymbol{H}=\left(\begin{array}{cccc}
\mathrm{e}^{\lambda} & 0 & \lambda-1 & \lambda \\
\lambda-1 & \lambda & \mathrm{e}^{\lambda} & 0 \\
0 & \mathrm{e}^{\lambda} & \lambda & \lambda+1 \\
\lambda & \lambda+1 & 0 & \mathrm{e}^{\lambda}
\end{array}\right),
$$

and where $\hat{\psi}=\left(\hat{\psi}_{1}^{+}, \hat{\psi}_{1}^{-}, \hat{\psi}_{2}^{+}, \hat{\psi}_{2}^{-}\right)^{\mathrm{T}}$ gathers the inverse Hankel transforms of the previously introduced auxiliary functions defined by $(2.16 a, b)$. Specifically, we have

$$
\begin{gathered}
\hat{\psi}_{1}^{ \pm}(\lambda)=\int_{0}^{\infty} r \psi_{1}^{ \pm}(r) J_{1}(\lambda r) \mathrm{d} r=\left(\frac{1}{2} \pm h\right) \exp \left(-\lambda\left(\frac{1}{2} \pm h\right)\right), \\
\hat{\psi}_{2}^{ \pm}(\lambda)=\int_{0}^{\infty} r \psi_{2}^{ \pm}(r) J_{0}(\lambda r) \mathrm{d} r=\left(\frac{1}{\lambda}+\frac{1}{2} \pm h\right) \exp \left(-\lambda\left(\frac{1}{2} \pm h\right)\right),
\end{gathered}
$$


(a)

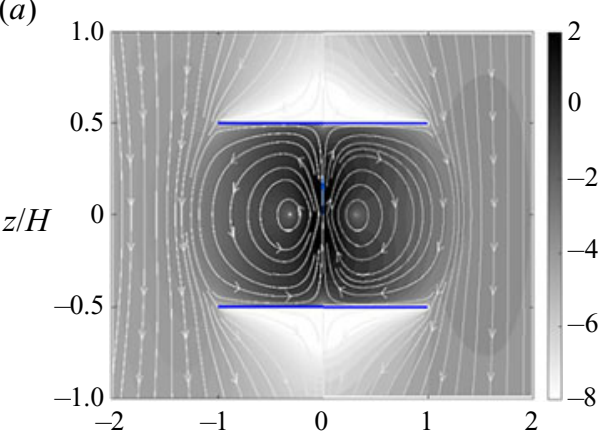

(c)

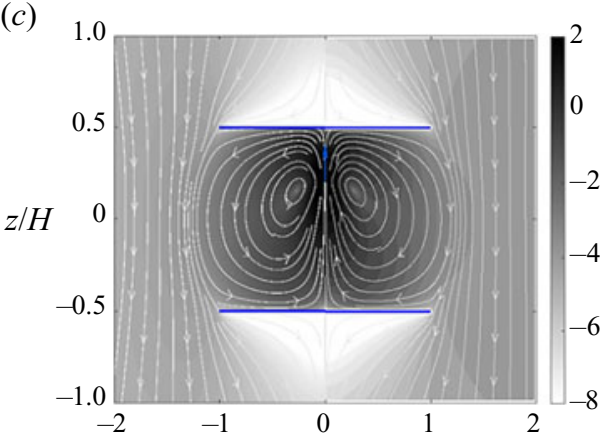

(e)

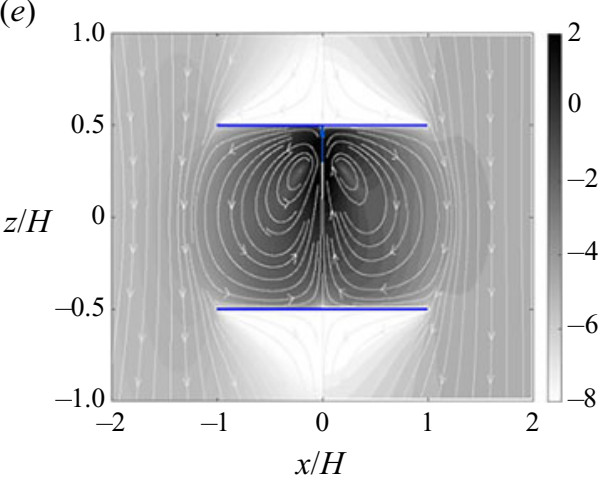

(b)

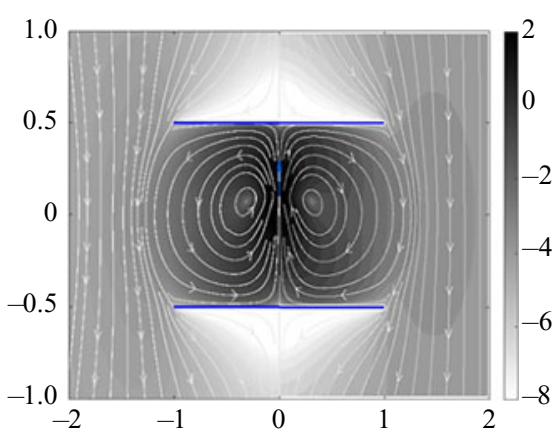

(d)

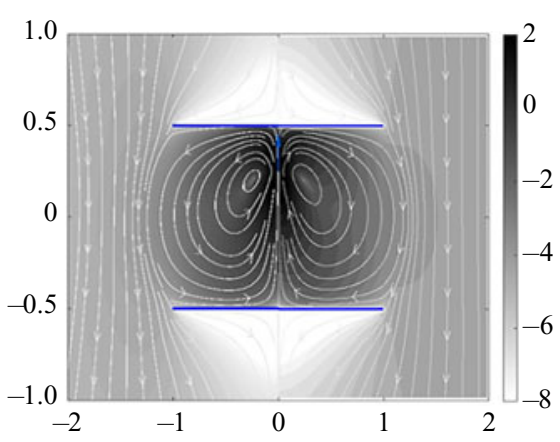

$(f)$

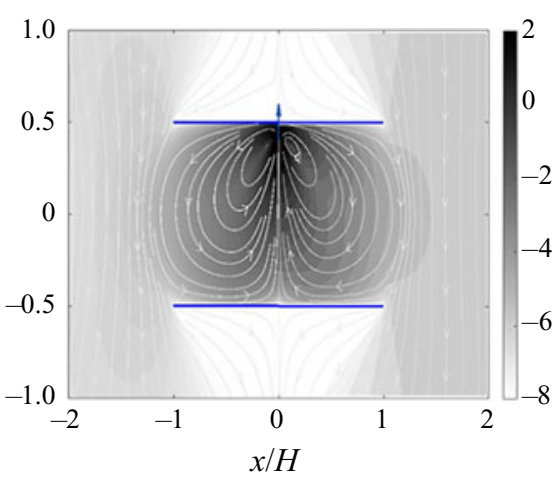

FIGURE 3. Streamlines and contour plots of the flow field induced by a point-force singularity acting inside two coaxially positioned disks of no-slip surfaces and of rescaled unit radius for various values of the vertical distance $h / H$. In each panel, the flow velocity field obtained using the present semi-analytical approach is displayed in the left domain corresponding to $x \leq 0$, while the solution obtained using FEM simulations is presented in the right domain corresponding to $x \geq 0$ for the same set of parameters. Here, we have defined the scaled flow velocity as $V=\boldsymbol{v} /(F /(8 \pi \eta))$. (a) $h / H=0,(b) h / H=0.1,(c) h / H=0.2,(d) h / H=0.25$, (e) $h / H=0.3,(f) h / H=0.4$.

for $|h|<1 / 2$. Solving the linear system of equations given by (2.25) and (2.26) for the unknown vector function $g$ upon making use of (2.13), (2.14) and (2.18) leads to

$$
X_{13}=\left(\mathrm{e}^{-\lambda h},-h \mathrm{e}^{-\lambda h}, \mathrm{e}^{\lambda h},-h \mathrm{e}^{\lambda h}\right)^{\mathrm{T}} .
$$


Accordingly, the total velocity and pressure fields in the lower and upper regions vanish in the limit $R \rightarrow \infty$. The corresponding solution in the intermediate fluid domain can readily be obtained by invoking (2.13) and (2.14).

\section{Hydrodynamic mobility}

Our calculation of the flow field presented in the previous section can be employed in order to probe the effect of the two hard disks on the hydrodynamic drag acting on an enclosed point-like particle axially moving along the coaxially positioned axis. This effect is commonly quantified by the hydrodynamic mobility function, which relates the velocity of a particle to the net force exerted on its surface (Leal 1980; Swan \& Brady 2007; Daddi-Moussa-Ider \& Gekle 2016, 2017, 2018; Driscoll \& Delmotte 2019). In a bulk Newtonian fluid of constant dynamic viscosity $\eta$, the mobility function $\mu$ of a spherical particle of radius $a$ is given by the familiar Stokes law (Stokes 1851), which states that in this case the mobility is $\mu_{0}=1 /(6 \pi \eta a)$. In the presence of the confining disks, the leading-order correction to the particle mobility for an axisymmetric motion along the axis is obtained by evaluating the image flow field at the particle position as

$$
\Delta \mu=F^{-1} \lim _{(r, z) \rightarrow(0, h)} v_{z 2}^{*}(r, z) .
$$

Evaluating the limit in the latter equation and scaling by the bulk mobility, the scaled correction to the particle mobility is obtained as

$$
\frac{\Delta \mu}{\mu_{0}}=-k a
$$

where

$$
k=\frac{3}{4} \int_{0}^{\infty}\left(\left((1-\lambda h) A_{2}^{+}-\lambda B_{2}^{+}\right) \mathrm{e}^{\lambda h}+\left((1+\lambda h) A_{2}^{-}+\lambda B_{2}^{-}\right) \mathrm{e}^{-\lambda h}\right) \mathrm{d} \lambda
$$

is a positive dimensionless number commonly denominated as the correction factor of the Stokes steady mobility (Happel \& Brenner 1983). Unfortunately, an analytical evaluation of this infinite integral is not auspicious. Therefore, we make recourse to a numerical evaluation.

For infinitely large disks, i.e. as $R \rightarrow \infty$, the correction factor $k$ in (3.2) can conveniently be cast into the simple integral form

$$
k_{\infty}=\frac{3}{8} \int_{0}^{\infty} W(\lambda)\left(\sinh ^{2} \lambda-\lambda^{2}\right)^{-1} \mathrm{~d} \lambda
$$

where we have defined the wavenumber-dependent function

$$
W(\lambda)=\Gamma_{+}+\Gamma_{-}+\gamma_{+}+\gamma_{-}+\mathrm{e}^{-2 \lambda}-\beta_{+} \beta_{-} \lambda^{3}-2 \lambda^{2}-2 \lambda-1,
$$

with

$$
\beta_{ \pm}=1 \pm 2 h, \quad \Gamma_{ \pm}=\left(1+\frac{1}{2} \lambda^{2} \beta_{ \pm}^{2}\right) \sinh \left(\lambda \beta_{\mp}\right), \quad \gamma_{ \pm}=\lambda \beta_{ \pm} \cosh \left(\lambda \beta_{\mp}\right) .
$$

This result is found to be in full agreement with the expression obtained by Swan \& Brady (2010), who used a two-dimensional Fourier transform technique. 


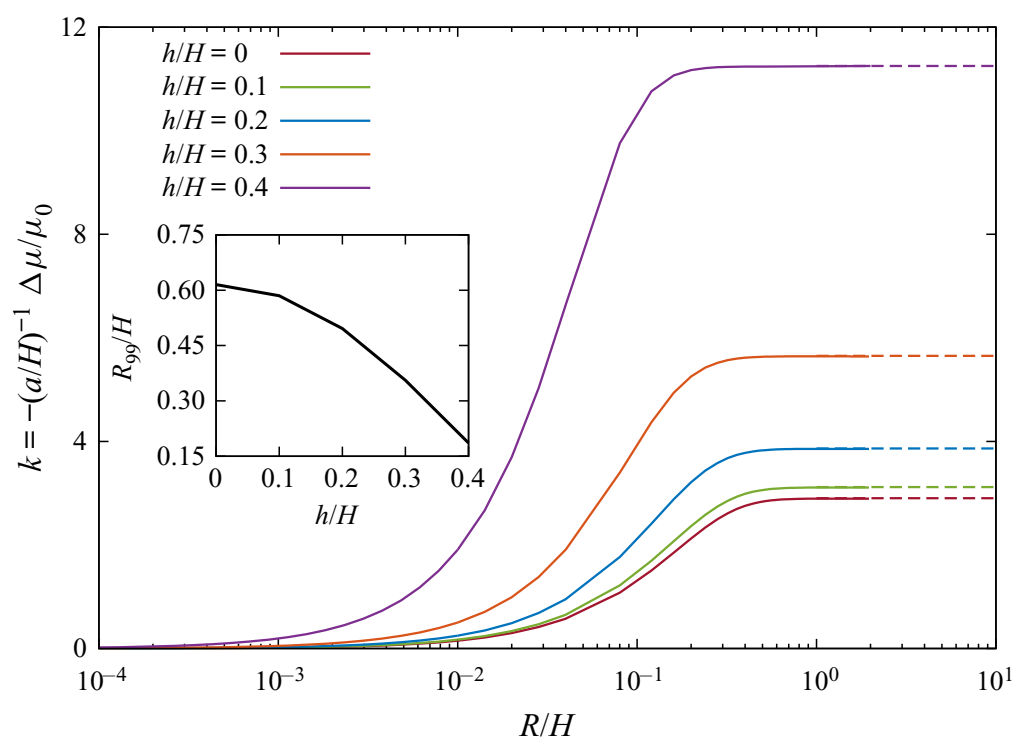

FIGURE 4. Variations of the correction factor of the hydrodynamic mobility as defined by (3.3) versus $R / H$ for various values of $h / H$. Horizontal dashed lines correspond to the correction factor near two infinitely large disks as given by (3.4). Inset: Evolution of $R_{99} / H$ versus $h / H$, where $R_{99}$ is defined such that $k\left(R_{99} / H\right)=0.99 k_{\infty}$, for which the correction factor near infinitely large disks is almost recovered.

In figure 4, we present a linear-logarithmic plot of the correction factor of the mobility function versus the radius of the disks for various values of the singularity position. Results are obtained by integrating (3.3) numerically. We observe that the curves follow a sigmoid-logistic-like phenomenology, implying that the correction factor increases significantly in the range of small radii before it reaches a saturation value. The latter corresponds to the correction factor predicted near two infinitely large disks given by (3.4).

Next, in order to quantify the effect of finite disk size on the correction to the hydrodynamic mobility, we customarily define the radius $R_{99}$ for which the mobility near infinitely large disks is essentially reached, such that $k\left(R_{99}\right)=0.99 k_{\infty}$. In the inset of figure 4, we display the variations of $R_{99}$ versus $h$ based on the data presented in the main plot. We observe that $R_{99}$ reaches a maximum value of approximately 0.62 at the mid-plane of the channel before it monotonically decreases with $h$. This observation suggests that, to a good approximation, the mobility near two infinitely large disks can adequately be used to estimate the mobility at an arbitrary position along the axis provided that the ratio of radius to channel height is above 0.62 . Hence, accounting for the finite-size effect here becomes crucial only for values below this threshold.

Finally, we comment on the applicability of the often-used approximation originally suggested by Oseen (1928) to predict the particle mobility between two boundaries by superimposing separately the leading-order effects of each boundary. Accordingly,

$$
\frac{\Delta \mu_{\text {Sup }}}{\mu_{0}}=-k_{\text {Sup }} a, \quad k_{\text {Sup }}=-a^{-1}\left(\left.\frac{\Delta \mu_{\text {Disk }}}{\mu_{0}}\right|_{b=1 / 2-h}+\left.\frac{\Delta \mu_{\text {Disk }}}{\mu_{0}}\right|_{b=1 / 2+h}\right),
$$

where the leading-order correction to the mobility function for axisymmetric motion normal to one rigid circular disk has previously been obtained by Kim (1983) and is 


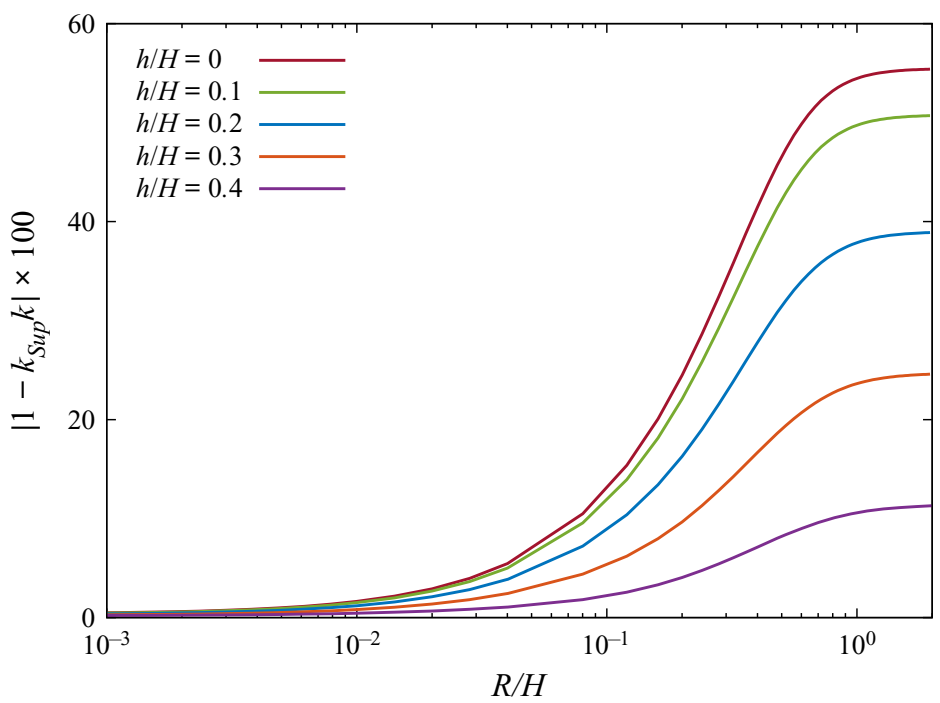

FIGURE 5. Percentage relative error between the correction factor of the Stokes steady mobility as obtained from the superposition approximation given by $(3.7 a, b)$ and the exact expression given by (3.3).

expressed by

$$
\frac{\Delta \mu_{\text {Disk }}}{\mu_{0}}=-\frac{3}{4 \pi}\left(\frac{3+5 \xi^{2}}{\left(1+\xi^{2}\right)^{2}}+\frac{3}{\xi} \arctan \left(\frac{1}{\xi}\right)\right) \frac{a}{R}
$$

wherein $\xi=b / R$ is a dimensionless parameter with $b$ denoting the distance between the particle and the centre of the disk. This solution was obtained by formulating the flow problem in terms of a mixed boundary-value problem and solving the resulting dual integral equations using an approach analogous to that employed in the present work. Notably, for $\xi \rightarrow 0$ we recover the familiar correction to the hydrodynamic mobility near an infinitely extended plane solid wall of no-slip boundary condition at its surface, namely $\Delta \mu_{\text {Disk }} / \mu_{0}=-9 a /(8 b)$, as originally obtained by Lorentz using the reciprocal theorem more than a century ago (Lorentz 1907; Lee, Chadwick \& Leal 1979).

We now assess the accuracy of the superposition approximation stated by $(3.7 a, b)$ by direct comparison with the exact prediction given by (3.3). In figure 5 , we plot the variations of the percentage relative error between the correction factors $k_{S u p}$ and $k$ versus the radius of the disks $R$ for various values of the particle position $h$. In the range of small values of $R$, the relative error amounts to small values, typically smaller than $10 \%$ for $R<0.1$. Upon increasing $R$, the relative error gradually increases in a logistic-like manner, before it saturates on a plateau value as $R$ gets larger. The maximum error is obtained for the particle located on the mid-plane between the two disks for $h=0$ and is found to be approximately $55 \%$ in the limit of infinite disk radius. Therefore, the superposition approximation cannot be applied properly in this case. Nonetheless, as the particle position gets closer to either disk, the maximum error notably decreases to amount to only approximately $12 \%$ for $h=0.4$. Consequently, the superposition approximation can frequently be utilised in this range of values to predict the hydrodynamic mobility for axisymmetric motion along the axis of the disks. 


\section{Conclusions}

To summarise, we have examined the axisymmetric Stokes flow resulting from a stokeslet singularity acting on the axis of two coaxially positioned circular disks of equal radius. We have formulated the solution for the viscous incompressible flow field as a mixed boundary-value problem, which we have then reduced to a system of dual integral equations for four unknown wavenumber-dependent functions. Most importantly, we have shown the existence of viscous toroidal eddies in the fluid region bounded by the two plates. In the limit of infinitely large disks, we have successfully recovered the classic solution by Liron \& Mochon (1976) for a stokeslet acting normal to two parallel planar walls.

Additionally, we have provided an integral expression of the hydrodynamic mobility function quantifying the effect of the confining plates on the motion of a point-like particle moving along the axis of the coaxially positioned disks. Furthermore, we have demonstrated that accounting for the finite-size effect of the disks becomes essential only below a threshold value of the ratio of radius to channel height. Beyond this value, the mobility near two infinitely large disks can appropriately be employed. Finally, we have tested the validity and robustness of Oseen's approximation that postulates that the particle mobility between two boundaries could approximately be predicted by superimposing the contributions from each boundary independently. We have found that this simplistic approximation works quite well as the particle gets closer to either boundary but severely breaks down when the particle is located in the mid-plane between the two disks.

The analytical approach in the present paper is based on the assumption of flow axisymmetry. The Stokes flow induced by a stokeslet directed along an arbitrary direction in the presence of two coaxially positioned disks would be worth investigating in a future study. We conjecture that this solution might be obtained by making use of the Green and Neumann functions supplemented by the edge function, following the approach by Miyazaki (1984). This solution can then be employed to evaluate the translational and rotational mobility functions of particles located at arbitrary positions between the two disks. Alternatively, the problem can possibly be approached differently by means of multipole expansion methods involving the expression of the relevant hydrodynamic fields using oblate spheroidal coordinates (Lee \& Leal 1980). This approach has been widely employed in the context of micromechanics of heterogeneous composite materials and fracture analysis (Kushch \& Sangani 2000; Kushch 2013). In principle, our calculations can be extended to account for higher-order correction factors in the aspect ratio between the radius of the disks and the distance between the particle and the bounding plates (Swan \& Brady 2010), but this would require a very challenging effort.

For applications requiring the precise manipulation of single molecules at the nanoscale level, the no-slip boundary condition may need to be lifted. In this context, the effect of partial slip at the surfaces of the disks is commonly characterised by assuming that the velocity components of the fluid tangent to the surfaces of the disks is proportional to the rate of strain at the surfaces (Lauga \& Squires 2005; Lasne et al. 2008). This is an interesting aspect that could be included in our formalism and represents a worthwhile extension of the problem for future studies. We hope that our study will prove useful to researchers as well as practitioners working on particulate flow problems involving finitely sized boundaries, and pave the way towards better design and control of various processes in micro- and nanofluidic systems. 


\section{Acknowledgements}

A.D.M.I., H.L. and A.M.M. gratefully acknowledge support from the DFG (Deutsche Forschungsgemeinschaft) through the projects DA 2107/1-1, LO 418/16-3 and ME $3571 / 2-2$.

\section{Declaration of interests}

The authors report no conflict of interest.

\section{Appendix A. Analytical expressions for the kernel functions}

In this appendix, we provide technical details regarding the analytical derivation of the kernel functions appearing in the system of Fredholm integral equations of the first kind given by (2.22) of the main body of the paper.

The kernel functions can be expressed as infinite integrals over the wavenumber $\lambda$ as

$$
\begin{gathered}
L_{1}(r, t)=\int_{0}^{\infty} \lambda^{1 / 2} \mathrm{e}^{-\lambda} J_{1}(\lambda r) J_{1 / 2}(\lambda t) \mathrm{d} \lambda, \\
L_{2}(r, t)=\int_{0}^{\infty}\left(\lambda^{1 / 2}+\lambda^{-1 / 2}\right) \mathrm{e}^{-\lambda} J_{0}(\lambda r) J_{1 / 2}(\lambda t) \mathrm{d} \lambda, \\
L_{3}(r, t)=\int_{0}^{\infty} \lambda^{1 / 2} \mathrm{e}^{-\lambda} J_{0}(\lambda r) J_{3 / 2}(\lambda t) \mathrm{d} \lambda, \\
L_{4}(r, t)=\int_{0}^{\infty}\left(\lambda^{1 / 2}-\lambda^{-1 / 2}\right) \mathrm{e}^{-\lambda} J_{1}(\lambda r) J_{3 / 2}(\lambda t) \mathrm{d} \lambda, \\
L_{5}(r, t)=\int_{0}^{\infty} \lambda^{-1 / 2} J_{1}(\lambda r) J_{3 / 2}(\lambda t) \mathrm{d} \lambda, \\
L_{6}(r, t)=\int_{0}^{\infty} \lambda^{-1 / 2} J_{0}(\lambda r) J_{1 / 2}(\lambda t) \mathrm{d} \lambda,
\end{gathered}
$$

where $(r, t) \in[0, R]^{2}$. It can be shown that the first four integrals can conveniently be expressed in closed mathematical forms as

$$
\begin{gathered}
L_{1}(r, t)=\left(\frac{2}{\pi t}\right)^{1 / 2} \frac{1}{r} \operatorname{Im}\left(\xi_{+} \delta_{+}\right), \\
L_{2}(r, t)=\left(\frac{2}{\pi t}\right)^{1 / 2}\left(\operatorname{Re}(\Lambda)+\operatorname{Im}\left(\delta_{-}\right)\right), \\
L_{3}(r, t)=\left(\frac{2}{\pi t}\right)^{1 / 2} \operatorname{Re}\left(\Lambda t^{-1}-\delta_{-}\right), \\
L_{4}(r, t)=\left(\frac{2}{\pi t}\right)^{1 / 2}\left(\operatorname{Re}\left(\chi_{1}\right)+\operatorname{Im}\left(\chi_{2}\right)\right),
\end{gathered}
$$


where we have defined the abbreviations

$$
\begin{aligned}
& \xi_{ \pm}=1 \pm \mathrm{i} t, \quad \delta_{ \pm}=\left(r^{2}+\xi_{ \pm}^{2}\right)^{-1 / 2}, \quad \Lambda=\arcsin \left(\frac{t+\mathrm{i}}{r}\right), \quad \sigma=\frac{r}{\xi_{-}+\delta_{-}^{-1}}, \\
& \alpha=\frac{t}{r}, \quad \chi_{1}=\delta_{-}\left(\frac{r}{2}\left(1+\sigma^{2}\right)+\frac{\xi_{-}}{r}\right)-\frac{\Lambda}{2 \alpha}, \quad \chi_{2}=\frac{1}{r t \delta_{-}}+\frac{\delta_{-}}{8 \alpha}\left(\xi_{-}-r \sigma^{3}\right) .
\end{aligned}
$$

In addition, the integrals $L_{5}$ and $L_{6}$ have analytical forms and can be calculated directly from standard integration tables or software algebra systems such as Mathematica (Wolfram 1999) as

$$
\begin{gathered}
L_{5}(r, t)=\frac{1}{2}\left(\frac{\pi}{2 t}\right)^{1 / 2} \alpha^{-1} H(t-r)+\left(\frac{1}{2 \pi t}\right)^{1 / 2}\left(\alpha^{-1} \arcsin (\alpha)-\left(1-\alpha^{2}\right)^{1 / 2}\right) H(r-t), \\
L_{6}(r, t)=\left(\frac{\pi}{2 t}\right)^{1 / 2} H(t-r)+\left(\frac{2}{\pi t}\right)^{1 / 2} \arcsin (\alpha) H(r-t),
\end{gathered}
$$

where $H(\cdot)$ denotes the Heaviside step function.

In the following, we will show how the integrals given by (A 1) can be evaluated analytically. The core idea of our approach consists of expressing these integrals in the form of Laplace transforms of Bessel functions of the first kind (Spiegel 1965; Widder 2015),

$$
\mathcal{L}\left\{J_{k}(z)\right\}(p)=\left(1+p^{2}\right)^{-1 / 2}\left(p+\left(1+p^{2}\right)^{1 / 2}\right)^{-k},
$$

and using the recurrence relation (Abramowitz \& Stegun 1972)

$$
\frac{2 k}{z} J_{k}(z)=J_{k-1}(z)+J_{k+1}(z) .
$$

In addition, we will employ the following identities providing closed-form expressions for the Bessel functions of the first kind of half-integer order in terms of the standard trigonometric functions,

$$
\begin{aligned}
J_{1 / 2}(z) & =\left(\frac{2}{\pi z}\right)^{1 / 2} \sin (z), \\
J_{-1 / 2}(z) & =\left(\frac{2}{\pi z}\right)^{1 / 2} \cos (z) .
\end{aligned}
$$

\section{A.1. Evaluation of the integral $L_{1}$}

By making use of the identity given by (A $7 a$ ), the integral $L_{1}$ stated by (A $\left.1 a\right)$ can be expressed as

$$
L_{1}(r, t)=\left(\frac{2}{\pi t}\right)^{1 / 2} \int_{0}^{\infty} \mathrm{e}^{-\lambda} J_{1}(\lambda r) \sin (\lambda t) \mathrm{d} \lambda .
$$

Using the change of variable $x=\lambda r$ and Euler's representation of the sine function, the latter integral can be expressed as

$$
L_{1}(r, t)=\left(\frac{2}{\pi t}\right)^{1 / 2} \frac{1}{r} \operatorname{Im}\left(\int_{0}^{\infty} \exp \left(-\frac{x}{r}(1-\mathrm{i} t)\right) J_{1}(x) \mathrm{d} x\right) .
$$


This leads to (A 2a) after making use of the Laplace transform given by (A 5) for $k=1$ and $p=(1-\mathrm{i} t) / r$. We note that $\operatorname{Im}(z)=-\operatorname{Im}(\bar{z})$ for $z \in \mathbb{C}$, where $\bar{z}$ denotes the complex conjugate of $z$.

\section{A.2. Evaluation of the integral $L_{2}$}

We next consider the integral defined by (A $1 b)$, which can conveniently be decomposed into two parts as

$$
L_{2}(r, t)=L_{2,1}(r, t)+L_{2,2}(r, t),
$$

where

$$
\begin{gathered}
L_{2,1}(r, t)=\left(\frac{2}{\pi t}\right)^{1 / 2} \int_{0}^{\infty} \mathrm{e}^{-\lambda} J_{0}(\lambda r) \sin (\lambda t) \mathrm{d} \lambda, \\
L_{2,2}(r, t)=\left(\frac{2}{\pi t}\right)^{1 / 2} \int_{0}^{t} \mathrm{~d} u \int_{0}^{\infty} \mathrm{e}^{-\lambda} J_{0}(\lambda r) \cos (\lambda u) \mathrm{d} \lambda .
\end{gathered}
$$

Here, we have made use of (A 7a) together with the integral representation

$$
\sin (\lambda t)=\lambda \int_{0}^{t} \cos (\lambda u) \mathrm{d} u .
$$

Using Euler's relation together with (A 5) for $k=0$, (A 11) can be evaluated as

$$
\begin{gathered}
L_{2,1}(r, t)=\left(\frac{2}{\pi t}\right)^{1 / 2} \operatorname{Im}\left(\left(r^{2}+(1-\mathrm{i} t)^{2}\right)^{-1 / 2}\right), \\
L_{2,2}(r, t)=\left(\frac{2}{\pi t}\right)^{1 / 2} \operatorname{Re}\left(\int_{0}^{t}\left(r^{2}+(1-\mathrm{i} u)^{2}\right)^{-1 / 2} \mathrm{~d} u\right) .
\end{gathered}
$$

The definite integral in (A 13b) can be evaluated as

$$
L_{2,2}(r, t)=\left(\frac{2}{\pi t}\right)^{1 / 2} \operatorname{Re}\left(\arcsin \left(\frac{t+\mathrm{i}}{r}\right)\right) .
$$

Equation (A 2b) follows forthwith after collecting terms.

It is worth mentioning that, for a given complex number $z=x+\mathrm{i} y$, the arcsine function is defined when $\pm x \notin(1, \infty)$ as (Abramowitz \& Stegun 1972)

$$
\arcsin (z)=\arcsin \left(\alpha_{-}\right)+\mathrm{i} \operatorname{sign}(y) \ln \left(\alpha_{+}+\left(\alpha_{+}^{2}-1\right)^{1 / 2}\right),
$$

where

$$
\alpha_{ \pm}=\frac{1}{2}\left((x+1)^{2}+y^{2}\right)^{1 / 2} \pm \frac{1}{2}\left((x-1)^{2}+y^{2}\right)^{1 / 2} .
$$


A.3. Evaluation of the integral $L_{3}$

Analogously, the integral $L_{3}$ defined by (A $1 c$ ) can be decomposed into two parts as

$$
L_{3}(r, t)=L_{3,1}(r, t)-L_{3,2}(r, t),
$$

upon using the recurrence relation stated by (A 6) and setting $k=1 / 2$ together with the identities given by (A 7). Here, we have defined $L_{3,1}(r, t)=t^{-1} L_{2,2}(r, t)$ and

$$
L_{3,2}(r, t)=\left(\frac{2}{\pi t}\right)^{1 / 2} \int_{0}^{\infty} \mathrm{e}^{-\lambda} J_{0}(\lambda r) \cos (\lambda t) \mathrm{d} \lambda,
$$

which can readily be evaluated as (A 11a) but this time by taking the real part. This leads to (A 2c) upon collecting terms.

\section{A.4. Evaluation of the integral $L_{4}$}

Finally, upon using (A 6) for $k=1 / 2$ and the identities given by (A 7), the integral $L_{4}$ can be decomposed into four parts:

$$
L_{4}(r, t)=L_{4,1}+L_{4,2}-\left(L_{4,3}+L_{4,4}\right),
$$

where we have defined

$$
\begin{gathered}
L_{4,1}(r, t)=\left(\frac{2}{\pi t}\right)^{1 / 2} t^{-1} \int_{0}^{\infty} \lambda^{-1} \mathrm{e}^{-\lambda} J_{1}(\lambda r) \sin (\lambda t) \mathrm{d} \lambda \\
L_{4,2}(r, t)=\left(\frac{2}{\pi t}\right)^{1 / 2} \int_{0}^{\infty} \lambda^{-1} \mathrm{e}^{-\lambda} J_{1}(\lambda r) \cos (\lambda t) \mathrm{d} \lambda \\
L_{4,3}(r, t)=\left(\frac{2}{\pi t}\right)^{1 / 2} \int_{0}^{\infty} \mathrm{e}^{-\lambda} J_{1}(\lambda r) \cos (\lambda t) \mathrm{d} \lambda \\
L_{4,4}(r, t)=\left(\frac{2}{\pi t}\right)^{1 / 2} t^{-1} \int_{0}^{\infty} \lambda^{-2} \mathrm{e}^{-\lambda} J_{1}(\lambda r) \sin (\lambda t) \mathrm{d} \lambda
\end{gathered}
$$

In the following, we will make use when appropriate of the shorthand notation defined in (A 3a). By using the integral representation of the sine function given by (A 12), the first integral can be expressed as

$$
L_{4,1}(r, t)=\left(\frac{2}{\pi t}\right)^{1 / 2} t^{-1} \int_{0}^{t} \mathrm{~d} u \int_{0}^{\infty} \mathrm{e}^{-\lambda} J_{1}(\lambda r) \cos (\lambda u) \mathrm{d} \lambda .
$$

Similarly, the evaluation of the indefinite integral over $\lambda$ can be performed using the Laplace transform of the Bessel function given by (A 5) to obtain

$$
L_{4,1}(r, t)=\left(\frac{2}{\pi t}\right)^{1 / 2}(t r)^{-1} \operatorname{Re}\left(\int_{0}^{t}\left(1-(1-\mathrm{i} u)\left(r^{2}+(1-\mathrm{i} u)^{2}\right)^{-1 / 2}\right) \mathrm{d} u\right) .
$$

The definite integral in the latter equation can then be evaluated and cast in the final simplified form

$$
L_{4,1}(r, t)=\left(\frac{2}{\pi t}\right)^{1 / 2} r^{-1}\left(1+t^{-1} \operatorname{Im}\left(\delta_{-}^{-1}\right)\right) .
$$

Next, the evaluation of the second integral is straightforward after expressing the first-order Bessel function as a function of the zeroth- and second-order Bessel functions 
using the recurrence relation given by (A 6) for $k=1$ to obtain

$$
L_{4,2}(r, t)=r(2 \pi t)^{-1 / 2} \int_{0}^{\infty} \mathrm{e}^{-\lambda}\left(J_{0}(\lambda r)+J_{2}(\lambda r)\right) \cos (\lambda t) \mathrm{d} \lambda,
$$

which can readily be evaluated as

$$
L_{4,2}(r, t)=r(2 \pi t)^{-1 / 2} \operatorname{Re}\left(\delta_{-}\left(1+\sigma^{2}\right)\right) .
$$

The third integral can be deduced from the calculation of $L_{1}(r, t)$ given by (A 9), this time by taking the real part to obtain

$$
L_{4,3}=\left(\frac{2}{\pi t}\right)^{1 / 2} r^{-1} \operatorname{Re}\left(1-\xi_{-} \delta_{-}\right) .
$$

Lastly, the fourth integral can be decomposed into two parts as

$$
L_{4,4}(r, t)=L_{4,4,1}(r, t)+L_{4,4,2}(r, t),
$$

where $L_{4,4,1}(r, t)=(2 \alpha)^{-1} L_{2,2}(r, t)$ and

$$
L_{4,4,2}(r, t)=(2 \pi t)^{-1 / 2} \frac{r}{t} \int_{0}^{\infty} \lambda^{-1} \mathrm{e}^{-\lambda} J_{2}(\lambda r) \sin (\lambda t) \mathrm{d} \lambda .
$$

This integral can be handled using the recurrence formula given by (A 6) to obtain

$$
L_{4,4,2}(r, t)=(2 \pi t)^{-1 / 2} \frac{r^{2}}{4 t} \int_{0}^{\infty} \mathrm{e}^{-\lambda}\left(J_{1}(\lambda r)+J_{3}(\lambda r)\right) \sin (\lambda t) \mathrm{d} \lambda .
$$

The latter integral can be calculated and cast in the final simplified form

$$
L_{4,4,2}(r, t)=(2 \pi t)^{-1 / 2}(4 \alpha)^{-1}\left(r \operatorname{Im}\left(\delta_{-} \sigma^{3}\right)-\operatorname{Im}\left(\xi_{-} \delta_{-}\right)\right) .
$$

By collecting terms, (A $2 d$ ) is readily obtained.

\section{REFERENCES}

Abramowitz, M. \& Stegun, I. A. 1972 Handbook of Mathematical Functions. Dover.

Asta, A. J., Palaia, I., Trizac, E., Levesque, M. \& Rotenberg, B. 2019 Lattice Boltzmann electrokinetics simulation of nanocapacitors. J. Chem. Phys. 151 (11), 114104.

Babel, S., Eikerling, M. \& Löwen, H. 2018 Impedance resonance in narrow confinement. J. Phys. Chem. C 122 (38), 21724-21734.

Balducci, A., Mao, P., Han, J. \& Doyle, P. S. 2006 Double-stranded DNA diffusion in slitlike nanochannels. Macromolecules 39 (18), 6273-6281.

BARON, M., BŁAWZDZIEWICZ, J. \& WAJNRYB, E. 2008 Hydrodynamic crystals: collective dynamics of regular arrays of spherical particles in a parallel-wall channel. Phys. Rev. Lett. 100 (17), 174502.

Bechinger, C., Di leonardo, R., Löwen, H., Reichhardt, C., Volpe, G. \& Volpe, G. 2016 Active particles in complex and crowded environments. Rev. Mod. Phys. 88 (4), 045006.

BECKER, R. \& BRAACK, M. 2001 A finite element pressure gradient stabilization for the Stokes equations based on local projections. Calcolo 38 (4), 173-199.

Benesch, T., Yiacoumi, S. \& Tsouris, C. 2003 Brownian motion in confinement. Phys. Rev. E 68 (2), 021401.

BHATTACHARYA, S. 2008 Cooperative motion of spheres arranged in periodic grids between two parallel walls. J. Chem. Phys. 128 (7), 074709. 
Bhattacharya, S. \& BŁawzDZiewicz, J. 2002 Image system for Stokes-flow singularity between two parallel planar walls. J. Math. Phys. 43 (11), 5720-5731.

Bhattacharya, S., BŁaWZdZiewicz, J. \& WajnRYB, E. 2005 Hydrodynamic interactions of spherical particles in suspensions confined between two planar walls. J. Fluid Mech. 541, 263-292.

Bhattacharya, S., BŁaWZdZiewicz, J. \& WajnRyB, E. 2006 Hydrodynamic interactions of spherical particles in Poiseuille flow between two parallel walls. Phys. Fluids 18 (5), 053301.

Bilbao, A., Wajnryb, E., Vanapalli, S. A. \& Beawzdziewicz, J. 2013 Nematode locomotion in unconfined and confined fluids. Phys. Fluids 25 (8), 081902.

Blake, J. R. 1971 A note on the image system for a Stokeslet in a no-slip boundary. Math. Proc. Camb. Phil. Soc. 70 (02), 303-310.

BŁAWZDZIEWICZ, J. \& WAJNRYB, E. 2008 An analysis of the far-field response to external forcing of a suspension in the Stokes flow in a parallel-wall channel. Phys. Fluids 20 (9), 093303.

BRAACK, M. \& RICHTER, T. 2006 Solutions of 3D Navier-Stokes benchmark problems with adaptive finite elements. Comput. Fluids 35 (4), 372-392.

BRACEWELL, R. 1999 The Fourier Transform and Its Applications. McGraw-Hill.

BRENNER, M. P. 1999 Screening mechanisms in sedimentation. Phys. Fluids 11 (4), 754-772.

Brotto, T., Caussin, J.-B., Lauga, E. \& Bartolo, D. 2013 Hydrodynamics of confined active fluids. Phys. Rev. Lett. 110 (3), 038101.

Campbell, L. C., Wilkinson, M. J., Manz, A., Camilleri, P. \& Humphreys, C. J. 2004 Electrophoretic manipulation of single DNA molecules in nanofabricated capillaries. Lab Chip 4 (3), 225-229.

Copson, E. T. 1961 On certain dual integral equations. Glasgow Math. J. 5 (1), 21-24.

Cross, J. D., Strychalski, E. A. \& Craighead, H. G. 2007 Size-dependent DNA mobility in nanochannels. J. Appl. Phys. 102 (2), 024701.

DAdDi-Moussa-Ider, A. \& GeKLE, S. 2016 Hydrodynamic interaction between particles near elastic interfaces. J. Chem. Phys. 145 (1), 014905.

DAdDi-Mouss A-IdER, A. \& GeKLE, S. 2017 Hydrodynamic mobility of a solid particle near a spherical elastic membrane: axisymmetric motion. Phys. Rev. E 95, 013108.

DAddi-Moussa-Ider, A. \& GeKLe, S. 2018 Brownian motion near an elastic cell membrane: a theoretical study. Eur. Phys. J. E 41 (2), 19.

Daddi-Moussa-Ider, A., Guckenberger, A. \& Gekle, S. 2016 Particle mobility between two planar elastic membranes: Brownian motion and membrane deformation. Phys. Fluids 28 (7), 071903.

Daddi-Moussa-Ider, A., Kaoui, B. \& Löwen, H. 2019 Axisymmetric flow due to a Stokeslet near a finite-sized elastic membrane. J. Phys. Soc. Japan 88 (5), 054401.

Daddi-Moussa-Ider, A., Lisicki, M., Löwen, H. \& Menzel, A. M. 2020 Dynamics of a microswimmer-microplatelet composite. Phys. Fluids 32 (2), 021902.

Daddi-Moussa-Ider, A., Lisicki, M., Mathijssen, A. J. T. M., Hoell, C., Goh, S., BŁawzdziewicz, J., Menzel, A. M. \& Löwen, H. 2018 State diagram of a three-sphere microswimmer in a channel. J. Phys.: Condens. Matter 30 (25), 254004.

Dai, L., Tree, D. R., van Der MaArel, J. R. C., Dorfman, K. D. \& Doyle, P. S. 2013 Revisiting blob theory for DNA diffusivity in slitlike confinement. Phys. Rev. Lett. 110, 168105.

Daiguji, H., Yang, P., Szeri, A. J. \& Majumdar, A. 2004 Electrochemomechanical energy conversion in nanofluidic channels. Nano Lett. 4 (12), 2315-2321.

Dalal, S., FARUtin, A. \& Misbah, C. 2020 Amoeboid swimming in compliant channel. Soft Matt. 16, 1599-1613.

Darwiche, A., Ingremeau, F., Amarouchene, Y., MaAli, A., Dufour, I. \& Kellay, H. 2013 Rheology of polymer solutions using colloidal-probe atomic force microscopy. Phys. Rev. E 87 (6), 062601.

DaVis, P. J. \& RABINOWITZ, P. 2007 Methods of Numerical Integration. Courier Corporation.

Doyle, P. S., Bibette, J., BAnCAud, A. \& Viovy, J.-L. 2002 Self-assembled magnetic matrices for DNA separation chips. Science 295 (5563), 2237-2237.

Driscoll, M. \& Delmotte, B. 2019 Leveraging collective effects in externally driven colloidal suspensions: experiments and simulations. Curr. Opin. Colloid Interface Sci. 40, $42-57$. 
Dufour, I., Mahli, A., Amarouchene, Y., Ayela, C., Caillard, B., Darwiche, A., Guirardel, M., Kellay, H., Lemaire, E., Mathieu, F., et al. 2012 The microcantilever: a versatile tool for measuring the rheological properties of complex fluids. J. Sens. 2012, 719898.

Dufresne, E. R., Altman, D. \& Grier, D. G. 2001 Brownian dynamics of a sphere between parallel walls. Europhys. Lett. 53 (2), 264.

Faucheux, L. P. \& Libchaber, A. J. 1994 Confined Brownian motion. Phys. Rev. E 49, 5158-5163.

FAXÉN, H. 1921 Einwirkung der Gefässwände auf den Widerstand gegen die Bewegung einer kleinen Kugel in einer zähen Flüssigkeit. PhD thesis, Uppsala University, Uppsala, Sweden.

FELDERHOF, B. U. 2006 Diffusion and velocity relaxation of a Brownian particle immersed in a viscous compressible fluid confined between two parallel plane walls. J. Chem. Phys. 124 (5), 054111.

FELDERHOF, B. U. 2010a Echoing in a viscous compressible fluid confined between two parallel plane walls. J. Fluid Mech. 656, 223-230.

FELDERHOF, B. U. $2010 b$ Loss of momentum in a viscous compressible fluid due to no-slip boundary condition at one or two planar walls. J. Chem. Phys. 133 (7), 074707.

François, N., Amarouchene, Y., Lounis, B. \& Kellay, H. 2009 Polymer conformations and hysteretic stresses in nonstationary flows of polymer solutions. Europhys. Lett. 86 (3), 34002.

François, N., Lasne, D., Amarouchene, Y., Lounis, B. \& Kellay, H. 2008 Drag enhancement with polymers. Phys. Rev. Lett. 100 (1), 018302.

Ganatos, P., Pfeffer, R. \& Weinbaum, S. 1980a A strong interaction theory for the creeping motion of a sphere between plane parallel boundaries. Part 2. Parallel motion. J. Fluid Mech. 99, 755-783.

Ganatos, P., Weinbaum, S. \& PfefFer, R. $1980 b$ A strong interaction theory for the creeping motion of a sphere between plane parallel boundaries. Part 1. Perpendicular motion. J. Fluid Mech. 99, 739-753.

Gompper, G., Winkler, R. G., Speck, T., Solon, A., Nardini, C., Peruani, F., Löwen, H., Golestanian, R., Kaupp, U. B., Alvarez, L., et al. 2020 The 2020 motile active matter roadmap. J. Phys.: Condens. Matter 32 (19), 193001.

GraHAM, M. D. 2011 Fluid dynamics of dissolved polymer molecules in confined geometries. Annu. Rev. Fluid Mech. 43, 273-298.

Griggs, A. J., Zinchenko, A. Z. \& DAVIS, R. H. 2007 Low-Reynolds-number motion of a deformable drop between two parallel plane walls. Intl J. Multiphase Flow 33 (2), 182-206.

Hackborn, W. W. 1990 Asymmetric Stokes flow between parallel planes due to a rotlet. J. Fluid Mech. 218, 531-546.

Happel, J. \& BRenner, H. 1983 Low Reynolds Number Hydrodynamics: With Special Applications to Particulate Media. Springer, Martinus Nijhoff Publishers, The Hague.

IMAI, I. 1973 Fluid Dynamics (Ryūtai Rikigaku). Syokabo Publishing. [in Japanese].

JANSSEN, P. J. A. \& Anderson, P. D. 2007 Boundary-integral method for drop deformation between parallel plates. Phys. Fluids 19 (4), 043602.

JANSSEN, P. J. A. \& Anderson, P. D. 2008 A boundary-integral model for drop deformation between two parallel plates with non-unit viscosity ratio drops. J. Comp. Phys. 227 (20), 8807-8819.

Jones, R. B. 2004 Spherical particle in Poiseuille flow between planar walls. J. Chem. Phys. 121 (1), 483-500.

Jones, J. J., VAn Der MAAREL, J. R. C. \& Doyle, P. S. 2013 Intrachain dynamics of large dsDNA confined to slitlike channels. Phys. Rev. Lett. 110 (6), 068101.

KIM, M. U. 1983 Axisymmetric Stokes flow due to a point force near a circular disk. J. Phys. Soc. Japan $52(2), 449-455$.

Kim, S. \& Karrila, S. J. 2013 Microhydrodynamics: Principles and Selected Applications. Courier Corporation.

KushCH, V. I. 2013 Micromechanics of Composites: Multipole Expansion Approach. Butterworth-Heinemann.

KUSHCH, V. I. \& SANGANI, A. S. 2000 Conductivity of a composite containing uniformly oriented penny-shaped cracks or perfectly conducting discs. Proc. R. Soc. Lond. A 456 (1995), 683-699.

Lasne, D., MaAli, A., Amarouchene, Y., Cognet, L., Lounis, B. \& Kellay, H. 2008 Velocity profiles of water flowing past solid glass surfaces using fluorescent nanoparticles and molecules as velocity probes. Phys. Rev. Lett. 100 (21), 214502. 
LaUga, E. 2016 Bacterial hydrodynamics. Annu. Rev. Fluid Mech. 48, 105-130.

Lauga, E., Brenner, M. \& Stone, H. 2007 Microfluidics: the no-slip boundary condition. In Springer Handbook of Experimental Fluid Mechanics (ed. C. Tropea, A. Yarin \& J. F. Foss), pp. 1219-1240. Springer.

Lauga, E. \& Powers, T. R. 2009 The hydrodynamics of swimming microorganisms. Rep. Prog. Phys. $72(9), 096601$.

LAUGA, E. \& SQUiReS, T. M. 2005 Brownian motion near a partial-slip boundary: a local probe of the no-slip condition. Phys. Fluids 17 (10), 103102.

Le Goff, A., Kaoui, B., Kurzawa, G., Haszon, B. \& Salsac, A.-V. 2017 Squeezing bio-capsules into a constriction: deformation till break-up. Soft Matt. 13 (41), 7644-7648.

LEAL, L. G. 1980 Particle motions in a viscous fluid. Annu. Rev. Fluid Mech. 12 (1), 435-476.

Lee, S. H., Chadwick, R. S. \& Leal, L. G. 1979 Motion of a sphere in the presence of a plane interface. Part 1. An approximate solution by generalization of the method of Lorentz. J. Fluid Mech. 93, 705-726.

LEE, S. H. \& LEAL, L. G. 1980 Motion of a sphere in the presence of a plane interface. Part 2. An exact solution in bipolar co-ordinates. J. Fluid Mech. 98, 193-224.

LIN, B., YU, J. \& RICE, S. A. 2000 Direct measurements of constrained Brownian motion of an isolated sphere between two walls. Phys. Rev. E 62, 3909-3919.

LiRON, N. 1978 Fluid transport by cilia between parallel plates. J. Fluid Mech. 86 (4), 705-726.

LiRon, N. \& Blake, J. R. 1981 Existence of viscous eddies near boundaries. J. Fluid Mech. 107, $109-129$.

Liron, N. \& Mochon, S. 1976 Stokes flow for a Stokeslet between two parallel flat plates. J. Engng Maths 10 (4), 287-303.

LOBRY, L. \& OSTROWSKY, N. 1996 Diffusion of Brownian particles trapped between two walls: theory and dynamic-light-scattering measurements. Phys. Rev. B 53, 12050-12056.

LorentZ, H. A. 1907 Ein allgemeiner Satz, die Bewegung einer reibenden Flüssigkeit betreffend, nebst einigen Anwendungen desselben. Abh. Theor. Phys. 1, 23.

Marini Bettolo Marconi, U. \& Melchionna, S. 2012 Charge transport in nanochannels: a molecular theory. Langmuir 28 (38), 13727-13740.

Mathijssen, A. J. T. M., Doostmohammadi, A., Yeomans, J. M. \& Shendruk, T. N. 2016 Hydrodynamics of microswimmers in films. J. Fluid Mech. 806, 35-70.

Menzel, A. M. 2013 Unidirectional laning and migrating cluster crystals in confined self-propelled particle systems. J. Phys.: Condens. Matter 25 (50), 505103.

Menzel, A. M. 2015 Tuned, driven, and active soft matter. Phys. Rep. 554, 1-45.

Mewis, J. \& Wagner, N. J. 2012 Colloidal Suspension Rheology. Cambridge University Press.

MIYAZAKI, T. 1984 The effect of a circular disk on the motion of a small particle in a viscous fluid. J. Phys. Soc. Japan 53 (3), 1017-1025.

Moffatt, H. K. 1964 Viscous and resistive eddies near a sharp corner. J. Fluid Mech. 18 (1), 1-18.

OsEen, C. W. 1928 Neuere Methoden und Ergebnisse in der Hydrodynamik. Leipzig, Akademische Verlagsgesellschaft, M. B. H.

Ostapenko, T., Schwarzendahl, F. J., Böddeker, T. J., Kreis, C. T., Cammann, J., Mazza, M. G. \& BÄUMCHEN, O. 2018 Curvature-guided motility of microalgae in geometric confinement. Phys. Rev. Lett. 120, 068002.

OZARKAR, S. S. \& SANGANI, A. S. 2008 A method for determining Stokes flow around particles near a wall or in a thin film bounded by a wall and a gas-liquid interface. Phys. Fluids 20 (6), 063301.

PARK, S.-Y. \& Dimitrakopoulos, P. 2013 Transient dynamics of an elastic capsule in a microfluidic constriction. Soft Matt. 9 (37), 8844-8855.

PERSSON, F. \& TEGENFELDT, J. O. 2010 DNA in nanochannels-directly visualizing genomic information. Chem. Soc. Rev. 39 (3), 985-999.

Polyanin, A. D. \& MAnZHIROV, A. V. 1998 Handbook of Integral Equations. CRC Press.

Probstein, R. F. 2005 Physicochemical Hydrodynamics: An Introduction. John Wiley \& Sons.

Reisner, W., Morton, K. J., Riehn, R., WAng, Y. M., Yu, Z., Rosen, M., Sturm, J. C., Chou, S. Y., Frey, E. \& Austin, R. H. 2005 Statics and dynamics of single DNA molecules confined in nanochannels. Phys. Rev. Lett. 94 (19), 196101. 
Richter, T. 2017 Fluid-structure Interactions: Models, Analysis and Finite Elements. Lecture Notes in Computational Science and Engineering, vol. 118. Springer.

Riehn, R., Lu, M., WAng, Y.-M., Lim, S. F., Cox, E. C. \& Austin, R. H. 2005 Restriction mapping in nanofluidic devices. Proc. Natl Acad. Sci. USA 102 (29), 10012-10016.

RoY, C. J. 2010 Review of discretization error estimators in scientific computing. In 48th AIAA Aerospace Sciences Meeting Including the New Horizons Forum and Aerospace Exposition, Orlando, Florida, p. 126.

Saintillan, D., Shaqfeh, E. S. G. \& Darve, E. 2006 Effect of flexibility on the shear-induced migration of short-chain polymers in parabolic channel flow. J. Fluid Mech. 557, 297-306.

Shaebani, M. R., Wysocki, A., Winkler, R. G., Gompper, G. \& Rieger, H. 2020 Computational models for active matter. Nat. Rev. Phys. 2, 181-199.

Smithies, F. 1958 Integral Equations. Cambridge University Press.

SNEDdon, I. N. 1960 The elementary solution of dual integral equations. Glasgow Math. J. 4 (3), 108-110.

SNEDDon, I. N. 1966 Mixed Boundary Value Problems in Potential Theory. North-Holland.

SPIEGEL, M. R. 1965 Laplace Transforms. McGraw-Hill.

Staben, M. E., Zinchenko, A. Z. \& Davis, R. H. 2003 Motion of a particle between two parallel plane walls in low-Reynolds-number Poiseuille flow. Phys. Fluids 15 (6), 1711-1733.

Stein, D., van der Heyden, F. H. J., Koopmans, W. J. A. \& DekKer, C. 2006 Pressure-driven transport of confined DNA polymers in fluidic channels. Proc. Natl Acad. Sci. USA 103 (43), $15853-15858$.

Stokes, G. G. 1851 On the effect of the internal friction of fluids on the motion of pendulums. Trans. Camb. Phil. Soc. 9, 8.

Strychalski, E. A., Levy, S. L. \& Craighead, H. G. 2008 Diffusion of DNA in nanoslits. Macromolecules 41 (20), 7716-7721.

SWAN, J. W. \& BRADY, J. F. 2007 Simulation of hydrodynamically interacting particles near a no-slip boundary. Phys. Fluids 19 (11), 113306.

SWAN, J. W. \& BRADY, J. F. 2010 Particle motion between parallel walls: hydrodynamics and simulation. Phys. Fluids 22 (10), 103301.

Swan, J. W. \& BRAdY, J. F. 2011 The hydrodynamics of confined dispersions. J. Fluid Mech. 687, 254-299.

Tang, J., Levy, S. L., Trahan, D. W., Jones, J. J., Craighead, H. G. \& Doyle, P. S. 2010 Revisiting the conformation and dynamics of DNA in slitlike confinement. Macromolecules 43 (17), 7368-7377.

THAKore, V. \& HickMAn, J. J. 2015 Charge relaxation dynamics of an electrolytic nanocapacitor. J. Phys. Chem. C 119 (4), 2121-2132.

TRÄNKLE, B., RUH, D. \& ROHRBACH, A. 2016 Interaction dynamics of two diffusing particles: contact times and influence of nearby surfaces. Soft Matt. 12 (10), 2729-2736.

Trégouët, C., Salez, T., MonteuX, C. \& Reyssat, M. 2018 Transient deformation of a droplet near a microfluidic constriction: a quantitative analysis. Phys. Rev. Fluids 3 (5), 053603.

Trégouët, C., Salez, T., Monteux, C. \& Reyssat, M. 2019 Microfluidic probing of the complex interfacial rheology of multilayer capsules. Soft Matt. 15 (13), 2782-2790.

TRICOMI, F. G. 1985 Integral Equations. Courier Corporation.

Turner, S. W., Perez, A. M., Lopez, A. \& Craighead, H. G. 1998 Monolithic nanofluid sieving structures for DNA manipulation. J. Vac. Sci. Technol. B 16 (6), 3835-3840.

UsPal, W. E., ERAL, H. B. \& DoYLE, P. S. 2013 Engineering particle trajectories in microfluidic flows using particle shape. Nat. Commun. 4, 2666.

WidDER, D. V. 2015 Laplace Transform (PMS-6). Princeton University Press.

Wolfram, S. 1999 The MATHEMATICA ${ }^{\circledR}$ Book, Version 4. Cambridge University Press.

Wu, H., Farutin, A., Hu, W.-F., Thiébaud, M., Rafaï, S., Peyla, P., Lai, M.-C. \& Misbah, C. 2016 Amoeboid swimming in a channel. Soft Matt. 12 (36), 7470-7484.

Wu, H., Thiébaud, M., Hu, W.-F., Farutin, A., Rafai, S., Lai, M.-C., Peyla, P. \& Misbah, C. 2015 Amoeboid motion in confined geometry. Phys. Rev. E 92 (5), 050701.

XIA, D., YAN, J. \& HOU, S. 2012 Fabrication of nanofluidic biochips with nanochannels for applications in DNA analysis. Small 8 (18), 2787-2801.

Zötth, A. \& StARK, H. 2016 Emergent behavior in active colloids. J. Phys.: Condens. Matter 28 (25), 253001. 